\title{
Recovery Act: Electrochromic Glazings Technology: Improved Performance, Lower Price
}

\section{Final Report}

Type of Report:

Principal Investigator

Program Director

Date Report Issued:

Period Covered

DOE Award Number:

Submitting Organization:
Final

Dr. Mark Burdis

Dr. Neil Sbar

$2^{\text {nd }}$ August 2012

$20^{\text {th }}$ July 2010 until 31 $1^{\text {st }}$ March 2012

DE-EE0003926

SAGE Electrochromics, Inc

One Sage Way

Faribault, MN 55021

507-331-4848

www.sage-ec.com

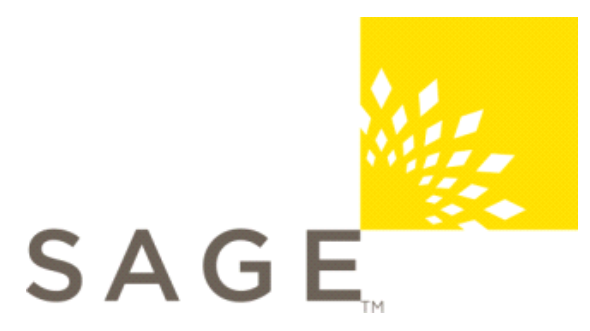




\section{Disclaimer:}

“This report was prepared as an account of work sponsored by an agency of the United States Government. Neither the United States Government nor any agency thereof, nor any of their employees, makes any warranty, express or implied, or assumes any legal liability or responsibility for the accuracy, completeness, or usefulness of any information, apparatus, product, or process disclosed, or represents that its use would not infringe privately owned rights. Reference herein to any specific commercial product, process, or service by trade name, trademark, manufacturer, or otherwise does not necessarily constitute or imply its endorsement, recommendation, or favoring by the United States Government or any agency thereof. The views and opinions of authors expressed herein do not necessarily state or reflect those of the United States Government or any agency thereof.” 


\section{Contents}

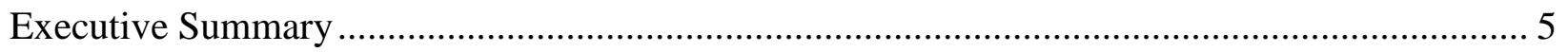

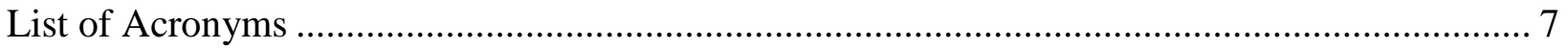

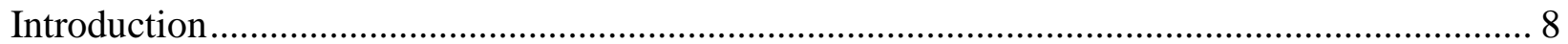

Project Description, Goals and Objectives ........................................................................... 8

Relationship to Announcement Objectives.......................................................................... 8

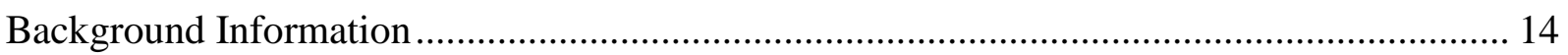

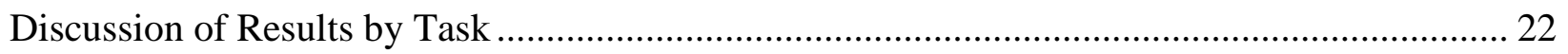

Task 2: Improve SHGC Range from 0.09 to 0.53 or Higher:..................................................... 22

Task 2.1: Replace the Bottom Transparent Conductor $\left(\mathrm{TC}_{\mathrm{b}}\right)$.............................................. 22

Task 2.1.1: Use Higher Transmittance Glass.................................................................... 27

Task 2.2: Replace Existing CE Material.......................................................................... 29

Task 2.3: Optimize Thin Film Stack................................................................................. 30

Task 3: Reduced Cost High Performance EC Windows .............................................................. 33

Task 3.1: Replace Lower Transparent Conductor ................................................................ 33

Task 3.2: Replace Thin Low-CTE Glass with Float Glass ...................................................... 34

Task 3.2.1: Develop an Effective Cleaning Process for Float Glass: ..................................... 35

Task 3.2.2: Determine if Mobile Cations Impact EC Device Performance............................ 37

Task 3.3: Increase the Sputter Rates for each of the EC Coatings ......................................... 38

Task 3.3.1: Target Material Improvements........................................................................... 38

Task 3.3.1.1: High Density Target Development .................................................................. 39

Task 3.3.1.2: Unique Dopant Target Development ............................................................... 42

Task 3.3.2: Understand Higher Deposition Rate Film Properties ......................................... 43

Task 3.4: Better Process Controls.................................................................................... 43

Task 3.4.1: Develop In-line Monitors for Critical Processes................................................... 44

Task 3.4.2: Develop Algorithms to Enable Closed-loop Control of the Process .................. 44

Task 4: Further Reduced Cost and Lower U-value ................................................................... 45

Task 4.1: Lower the sheet resistance of the top transparent conductor: ................................ 45

Task 4.1.1: Optimizing Optical Properties ............................................................................. 46

Task 4.1.2: Assure Compatibility with EC Device Layers....................................................... 46

Task 4.3: Determine U-values of Improved EC Windows .................................................... 46

Task 5: Ongoing Durability Testing of New/Improved Device Structures ................................ 47 


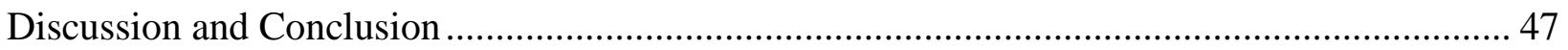

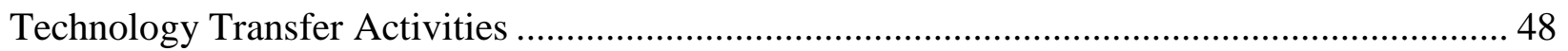

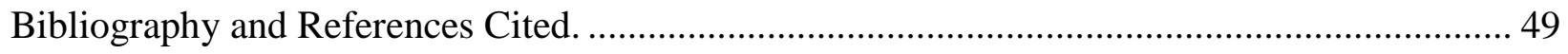




\section{Executive Summary}

The growing dependency of the US on energy imports and anticipated further increases in energy prices reinforce the concerns about meeting the energy demand in the future and one element of a secure energy future is conservation. It is estimated that the buildings sector represents $40 \%$ of the US's total energy consumption. And buildings produce as much as one third of the greenhouse gas emissions primarily through fossil fuel usage during their operational phase. A significant fraction of this energy usage is simply due to inefficient window technology.

Electrochromic (EC) windows allow electronic control of their optical properties so that the transparency to light can be adjusted from clear to dark. This ability to control the amount of solar energy allowed into the building can be advantageously used to minimize lighting, heating and air conditioning costs. Currently, the penetration of EC windows into the marketplace is extremely small, and consequently there is a huge opportunity for energy savings if this market can be expanded.

In order to increase the potential energy savings it is necessary to increase the quantity of EC windows in operation. Additionally, any incremental improvement in the energy performance of each window will add to the potential energy savings. The overall goals of this project were therefore to improve the energy performance and lower the cost of dynamic (EC) smart windows for residential and commercial building applications.

This project is obviously of benefit to the public by addressing two major areas: lowering the cost and improving the energy performance of EC glazings. The high level goals for these activities were: (i) to improve the range between the clear and the tinted state, (ii) reduce the price of EC windows by utilizing lower cost materials, (iii) lowering the U-Value ${ }^{1}$, and (iv) ensure the proposed changes have no detrimental effect to the proven durability of the window.

SAGE Electrochromics Inc. is the only company in the US which has a track record of producing EC windows, and presently has a small operational factory in Faribault MN which is shipping products throughout the world. There is a much larger factory currently under construction close by. This project was targeted specifically to address the issues outlined above, with a view to implementation on the new high volume manufacturing facility. Each of the Tasks which were addressed in this project is relatively straightforward to implement in this new facility and so the benefits of the work will be realized quickly.

The research described here has helped to understand and provide solutions to several interesting and previously unresolved issues of the technology as well as make progress in areas which will have a significant impact on energy saving. In particular several materials improvements have been made, and tasks related to throughput and yield improvements have been completed. All of this has been accomplished without any detrimental effect on the proven durability of the SageGlass EC device.

The project was divided into four main areas:

1. Improvement of the Properties of the EC device by material enhancements (Task 2);

2. Reduce the cost of production by improving the efficiency and yields of some key

\footnotetext{
${ }^{1}$ A measure $\left(\mathrm{Btu} / \mathrm{h} \mathrm{ft}{ }^{2} \mathrm{~F}\right)$ of how well a material or series of materials conducts heat. U-factors for window and door assemblies are the reciprocal of the assembly R-value: the smaller the U-factor, the less the heat flow.
} 
manufacturing processes (Task 3);

3. Further reduce the cost by significant modifications to the structure of the device (Task 4);

4. Ensure the durability of the EC device is not affected by any of the changes resulting from these activities (Task 5).

A detailed description of the activities carried out in these areas is given in the following report, along with the aims and goals of the work. We will see that we have completed Tasks 2 and 3 fully, and the durability of the resulting device structure has been unaffected. Some of Task 4 was not carried out because of difficulties with integrating the installation of the required targets into the production coater due to external constraints not related to this project. We will also see that the durability of the devices produced as a result of this work was largely unaffected by the changes made here, and so we can conclude that the objectives of Task 5 have also been fulfilled.

As a result, the future production of EC windows has benefited greatly from this work. We anticipate higher volumes and better yields for devices with an improved EC performance from the new high volume manufacturing facility currently under construction at SAGE. 


\section{List of Acronyms}

Ag - Silver

APCVD - Atmospheric Pressure Chemical Vapor Deposition

AZO - Aluminum doped Zinc Oxide

CDT - Current Device Technology

CE - Counter electrode

CTE - Coefficient of thermal expansion

CSSC - Chabot College Community and Student Services Center

DOE - Department of Energy

DOE - Design of Experiment

EC - Electrochromic

HIP - Hot isostatically pressed

HVAC - Heating Ventilation and Air Conditioning

HVM - High Volume Manufacturing

IC - Ion Conductor

IGU - Insulated Glazing Unit

ITO - Indium Tin Oxide

LBNL - Lawrence Berkeley National Laboratory

LCD - Liquid Crystal Display

LEED - Leadership in Energy and Environmental Design

NDT - New Device Technology

NREL - National Renewable Energy Laboratory

PNNL - Pacific Northwest National Laboratory

SHGC -Solar heat gain coefficient

SLG - Soda-lime glass

TC - Transparent Conductor

$\mathrm{TC}_{1}$ - Bottom (or lower) Transparent Conductor

$\mathrm{TC}_{2}$ - Top (or upper) Transparent Conductor

TEC - Transparent Electronic Conductor

$\mathrm{T}_{\text {vis }}$ - Visible light transmission

WWR - Window to wall ratio

ZEB - Zero energy buildings 


\section{Introduction}

\section{Project Description, Goals and Objectives}

The overall goals of this project were to improve the energy performance and lower the cost of dynamic electrochromic (EC) smart windows for residential and commercial building applications. This project was aligned with the Department of Energy (DOE) objective of zero energy buildings (ZEB) with high performance windows that provide thermal and daylighting benefits which can then be used to offset other building energy uses.

This project addressed two major areas: lowering the cost and improving the energy performance of EC glazings. The high level goals for these activities were: (i) to improve the SHGC ${ }^{2}$ range, (ii) reduce the price of EC windows by utilizing lower cost materials, (iii) lowering the UValue $^{3}$, and (iv) ensure the proposed changes have no detrimental effect to the proven durability of the window.

It was proposed to achieve these objectives using a combination of materials development and economies of scale facilitated by increased production volume. The materials developments were thought to be challenging but achievable, as will be demonstrated by the following narrative description, and the cost reductions were modeled on the adoption of low-e glass: a technology with a similar market structure. It will be shown that these objectives were, for the most part, successfully achieved.

\section{Relationship to Announcement Objectives}

Electrochromic windows have the potential to minimize lighting and HVAC use by altering the energy-load on the building in a controllable manner. Energy calculations comparing SAGE's EC windows with commercially available static glazings in the southwestern U.S. show average savings of $26 \%$ for a cooling dominated environment ${ }^{4}$. These and other simulations indicate that significant cumulative energy savings in the U.S. would be achieved if current static glazings were replaced by dynamic EC windows in a portion of commercial and residential buildings in both the new- and retrofit-markets. When included as part of a comprehensive building energy management strategy, EC windows can offer significant advantages over traditional static glazing. This project sought to accelerate market penetration of EC glazing by reducing the cost of EC technology, as well as improving the performance, thereby contributing to the DOE BTP net-zero energy building goals.

There is a need to improve the energy efficiency of building envelopes because they are the primary factor governing the heating, cooling, lighting and ventilation requirements of buildings, influencing $53 \%$ of building energy use ${ }^{5}$. In particular, windows contribute significantly to the overall energy performance of building envelopes. Thus there is a need to develop advanced energy efficient window glazing systems.

\footnotetext{
${ }^{2}$ The fraction of the sun's energy that is transmitted through the window, expressed in a number from zero to one. A window that has a SHGC of 0.3 will allow 30 percent of the sun's energy through.

${ }^{3} \mathrm{~A}$ measure $\left(\mathrm{Btu} / \mathrm{h} \mathrm{ft}^{2}{ }^{\circ} \mathrm{F}\right)$ of how well a material or series of materials conducts heat. U-factors for window and door assemblies are the reciprocal of the assembly R-value: the smaller the U-factor, the less the heat flow.

4 , E S. Energy Performance Analysis of Electrochromic Windows in New York Commercial Office Buildings. Lawrence Berkeley National Laboratory. Berkeley, CA. : s.n., 2002. LBNL-50096.

${ }^{5}$ Data contained in the NETLIII solicitation, 2001.
} 
It is therefore instructive to examine some examples of existing projects where SageGlass has been used to provide solutions to some of the issues addressed by this Department of Energy Initiative. These projects represent 'early adoption' by certain elements of the potential market, and it is anticipated that the work reported here will go some way to increasing the potential for market penetration, thereby improving overall energy performance of buildings in general.

SAGE Electrochromics has been shipping electrochromic windows since around 2004, and has completed several impressive projects throughout the world. An example of a project which uses EC glass to create a much more energy efficient building is that of Chabot College in California. When tBP/Architecture began work on the new Chabot College Community and Student Services Center (CSSC), the design team wanted to incorporate as much glass as possible to create a visually stunning centerpiece for the institution. They also wanted to preserve the view outside and flood the interior spaces with natural daylight. The CSSC is shown in Figure 1.

The CSSC atrium would include a two-story, 2,900-square-foot curtain wall facing south and west, posing significant glare and heat control challenges. Adding even greater complexity, Chabot College wanted the new CSSC to be an energy-efficient, LEED-certified building, part of a sustainability initiative calling for "green intelligent buildings" across campus.

Architects had to find a way to maintain outside views and keep people comfortable inside, while simultaneously minimizing energy consumption. Although conventional low-e glazing, automated interior blinds and automated exteriors louvers would manage the sun and solar heat gain, this approach would limit outside views and potentially compromise building aesthetics.

tBP/Architecture ultimately specified SageGlass ${ }^{\circledR}$ dynamic glazing for the new CSSC atrium. This solar control solution is cost-competitive with less efficient static approaches, which made it an easy decision for the project.

The SageGlass curtain wall features six zones integrated into the building management system. The glass darkens or clears by zone based on set air temperature thresholds, with a manual override for additional adjustments. Temperature is controlled by radiant heating and cooling in the concrete slab, combined with roof and ceiling air scoops for natural ventilation. No heating, ventilation and cooling (HVAC) system is required for the CSSC atrium, and the building qualifies for LEED certification.

Today, the CSSC serves as a thriving hub for nearly 15,000 Chabot College students, faculty and staff. SageGlass glazing keeps glare and temperature under control throughout the day, making effective use of the natural ventilation system. Sightlines to the surrounding campus grounds also remain unobstructed, while natural light reaches the interior spaces and reduces the need for artificial lighting.

According to Phil Newsom, tBP architect and senior project manager, SageGlass has made it possible to use an innovative natural air-cooling and heating strategy in the new building.

“The design approach we've taken for the CSSC delivers exceptional energy efficiency, due in large part to a ductless heating and cooling system that would not have been possible without deploying SageGlass,” Newsom said. 
"It's the architectural enabler that has allowed us to create a naturally ventilated, HVAC-free space." 


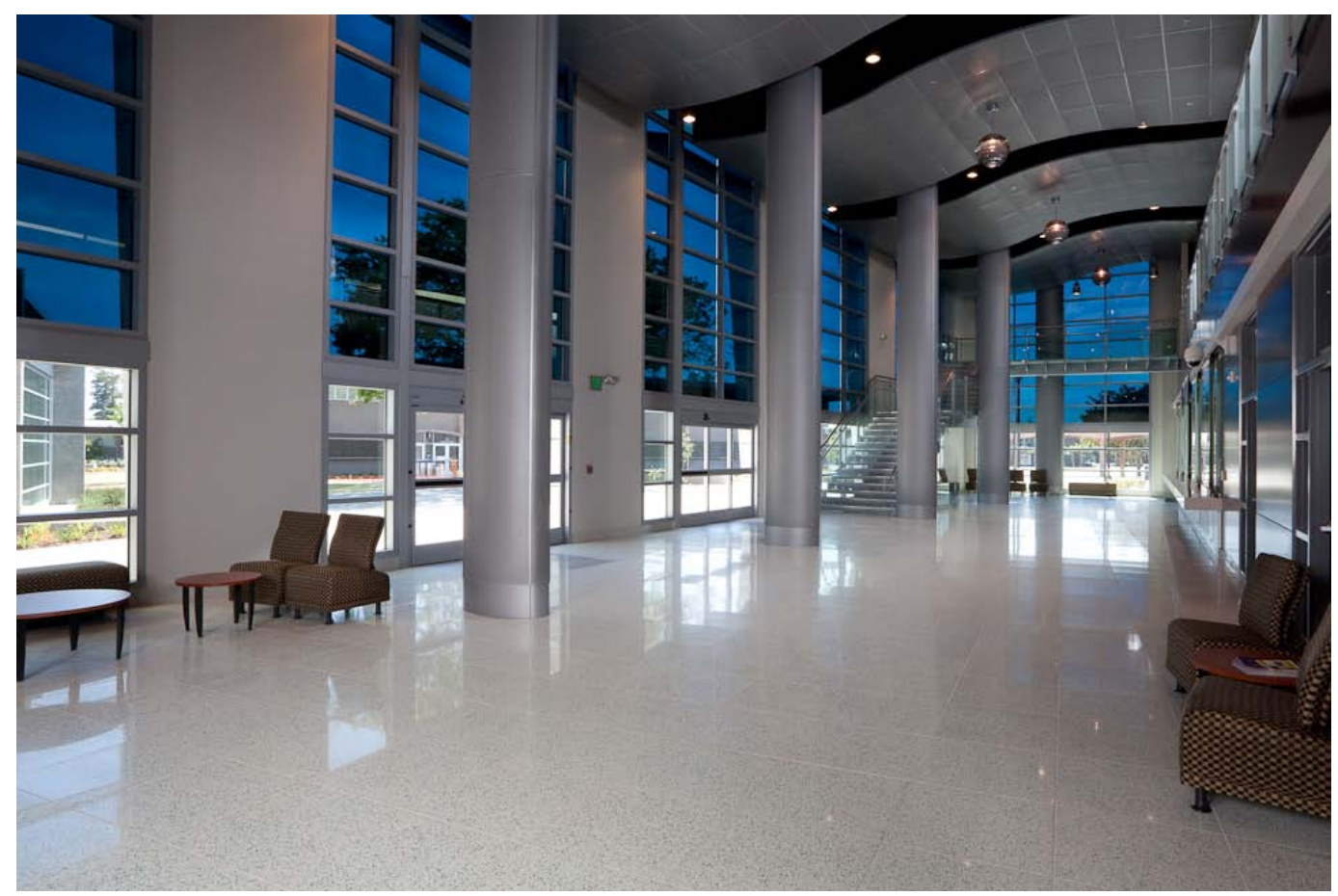

Figure 1 - An example of a electrochromic installation where the design of the building was influenced dramatically by the inclusion of EC in the project. In this case, the architect was able to modify the heating and cooling system to take account of the use of electrochromic technology, as well as modify the outside of the building as shown in the picture below.

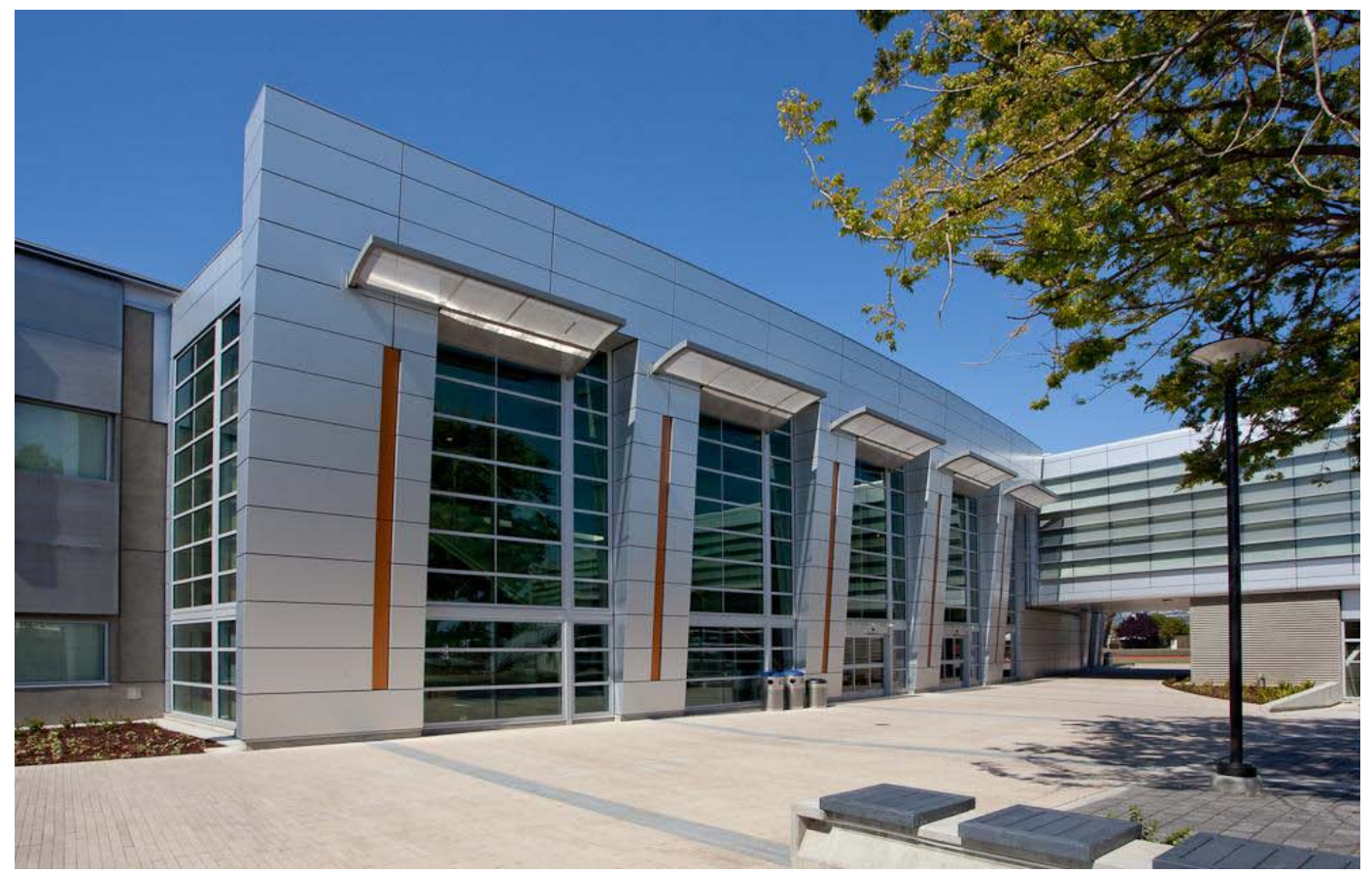


An additional benefit of electrochromic glass is the ability to block glare without losing the view of the outside, which is why windows are there in the first place. An excellent example of an application where this was the main driving force behind EC specification is described below.

Located near the Nebraska border in southeastern Wyoming, the Torrington Port of Entry serves as a busy weigh station at the intersection of U.S. Highways 26 and 85 . The interior of the facility is shown in Figure 2, where the upper picture shows the office with the glass in the tinted state, and the inset shows it in the clear state. Agents there promote traffic safety by monitoring commercial vehicles and ensuring compliance with state and federal laws. To best perform their jobs, the agents need clear and unobstructed views - from all angles, at all hours of the day, and in all weather conditions.

The existing weigh station had significant sun control issues. Intense sunlight and heat presented challenges during the summer, while snow glare added complexity in the winter. Baseball caps and sunglasses became mandatory accessories for agents monitoring traffic.

When hired to design a new Port of Entry facility, the architect knew these challenges could not be met with blinds, shades and other conventional sun controls, or with standard glazing by itself. They hired a daylighting consultant, Hutton Architecture Studio, to help him explore alternate glazings. It quickly became clear that SageGlass was the ideal solution.

In the Torrington facility, sensors are programmed to automatically let in one of four light levels through the glass throughout the day: $2 \%, 6 \%, 20 \%$, or $60 \%$ which was a feature critical to the visual success of the Port of Entry.

"SageGlass allowed the Wyoming Department of Transportation Port of Entry staff to have full view of trucks on the highway and those circulating through the Port without the need for interior blinds or exterior louvers on the windows to control summer and winter glare on the west and south elevations," said the Architect.

The new Torrington Port of Entry serves as a model weigh station for future facilities to follow. Dynamic SageGlass allows agents to clearly see out of the building at any time of day, without the need for interior blinds or exterior louvers to control summer and winter glare through windows facing west and south. Ball caps and sunglasses are no longer necessary.

It is also easier to maintain a constant, comfortable temperature inside throughout the year, and the facility now takes full advantage of natural daylight, improving energy efficiency by reducing dependence on artificial lighting.

"We are most pleased with the way SageGlass has performed in the building, and were pleasantly surprised at how easy the installation was," said Paul Hutton, president of Hutton Design Studio. "Most importantly, workers inside tell us they find the interior environment to be quite comfortable, promoting vehicle safety across the State of Wyoming.” 


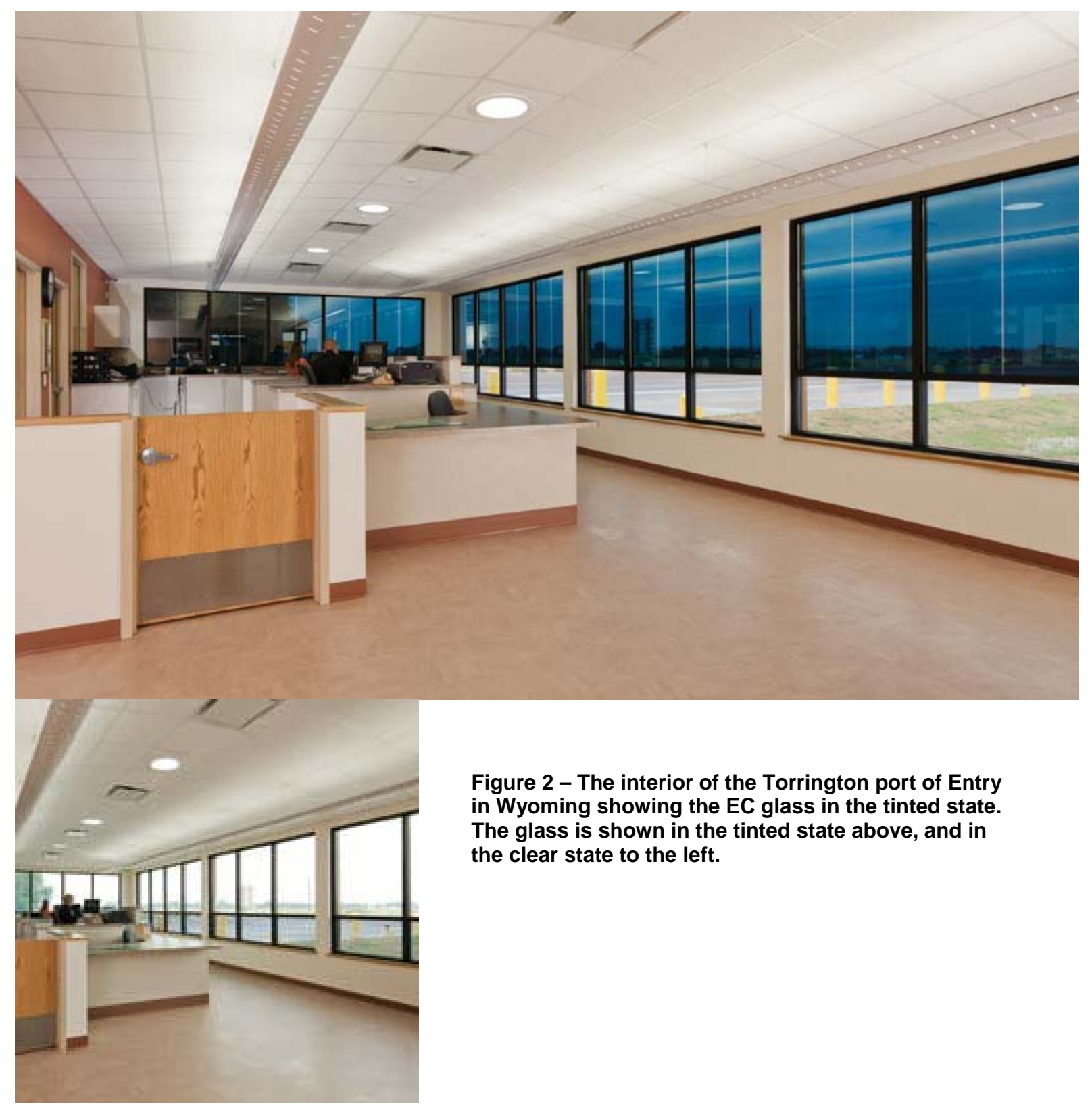

These two case studies serve to illustrate the potential which exists for energy saving throughout the US should EC technology achieve greater market penetration. The work done in this project will provide significant assistance in the pursuit of this aim. 


\section{Background Information}

In order to understand the progress and significance of the work described in this report, this section provides background and context. We begin with a description of the fundamental behavior of the electrochromic stack, and then discuss some of the areas which can benefit from

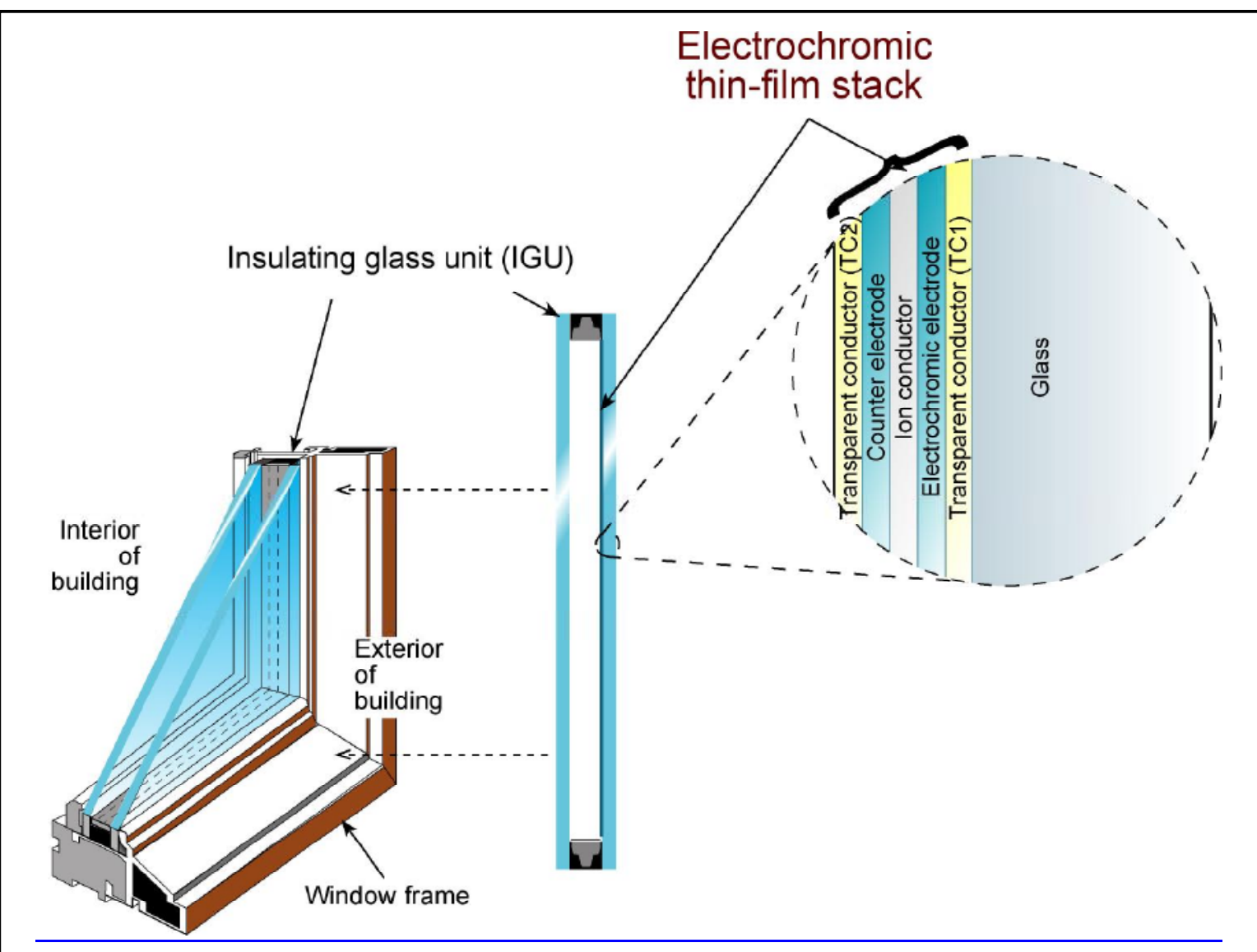

Figure 3. The EC thin-film stack is located on the inside surface of the outside pane of glass in a window, which means it blocks the near infra-red radiation (solar heat) before it can be transmitted through the glass.

work done as part of this project.

Electrochromic (EC) windows are glazings which can be tinted or cleared electronically to control solar heat and glare in buildings without blocking the view. They can be used to control the admission of sunlight into buildings to optimize the potential benefits of daylighting, as discussed in the first case study in the previous section, whilst maintaining outdoor views, and provide a higher degree of comfort while preventing glare, fading and overheating, as described in the second case study. By letting sunlight in on cool days and blocking it on hot days, EC windows can dramatically reduce energy demand. 
Another significant cost benefit of EC windows is the potential elimination of shades, blinds and louvers. EC glazings preserve views of the outdoors (the reason we have windows and skylights in the first place) and so can save cost by virtue of not requiring shades or blinds.

In order for the EC windows to be most effective in terms of energy savings, it is important that in the clear state the visible transmission is as transparent as possible, whilst in the tinted state it is as dark as possible. As will be shown, these objectives are often opposing, and so a series of decisions need to be made in order to achieve the best compromise. Additional important characteristics for EC windows are:

- Neutral color in both the clear and tinted state;

- Rapid, uniform switching between states;

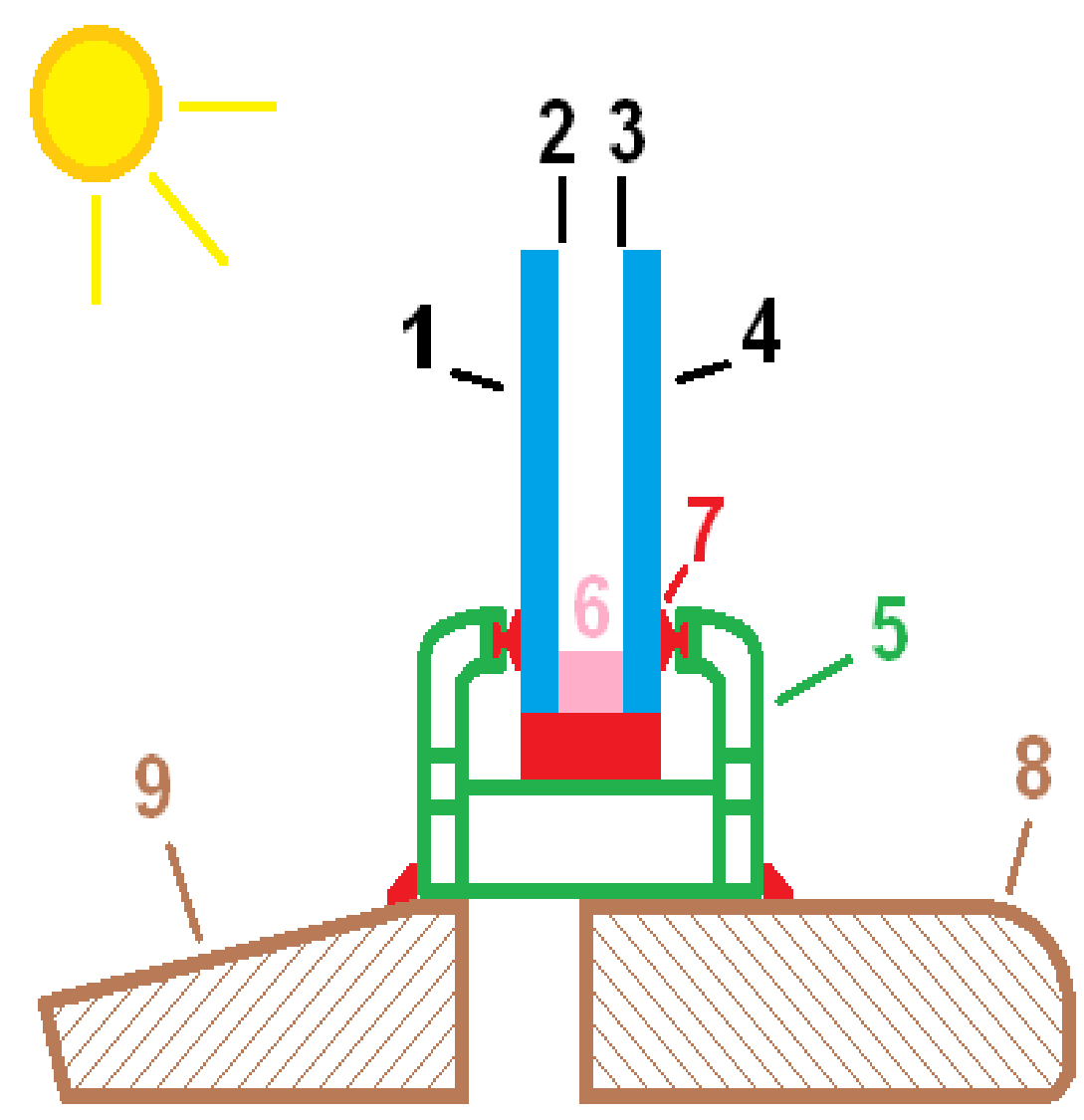

Figure 4 - A sectioned diagram of an Insulated Glazed Unit: a fixed double glazed window within a frame. Surface \#1 is facing outside, Surface \#2 is the inside surface of the exterior pane, Surface \#3 is the outside surface of the interior pane, and Surface \#4 is the inside surface of interior pane. The window frame is labeled \#5, a spacer is indicated as \#6, seals are shown in red (\#7), the internal reveal is on the right hand side (\#8) and the exterior windowsill on the left (\#9). (Image taken from Wikipedia: Author: NcLean).
- Low levels of reflection in both clear and tinted states;

- Absence of point defects;

- Low power and voltage requirements; and

- Low levels of electrical leakage current ${ }^{6}$.

Pursuit of these goals has led to the present state of the SageGlass ${ }^{\circledR}$ technology, and some early market penetration has taken place as a result. Further, more widespread penetration will require that the cost of the product is reduced without any compromise in the

\footnotetext{
${ }^{6}$ Leakage current is the equilibrium electronic current flowing through the device when the rate of change of coloration is zero.
} 
performance characteristics. Indeed, higher penetration would also be encouraged with any performance improvements.

Figure 3 shows the thin-film EC stack within the context of a typical window. This configuration allows the glass to be incorporated into a wide range of residential and commercial window systems with a minimum of disruption to existing framing systems. The only significant difference between these EC glazings and conventional glazings to the window manufacturer is the presence of a wire emerging from the edge of the frame and the subsequent issues with wire routing and power supplies for driving the windows.

In the example shown in Figure 3 the EC glazing, comprising a double glazed unit - also referred to as an insulated glazing unit (IGU) - is installed in a window frame with the coatings located

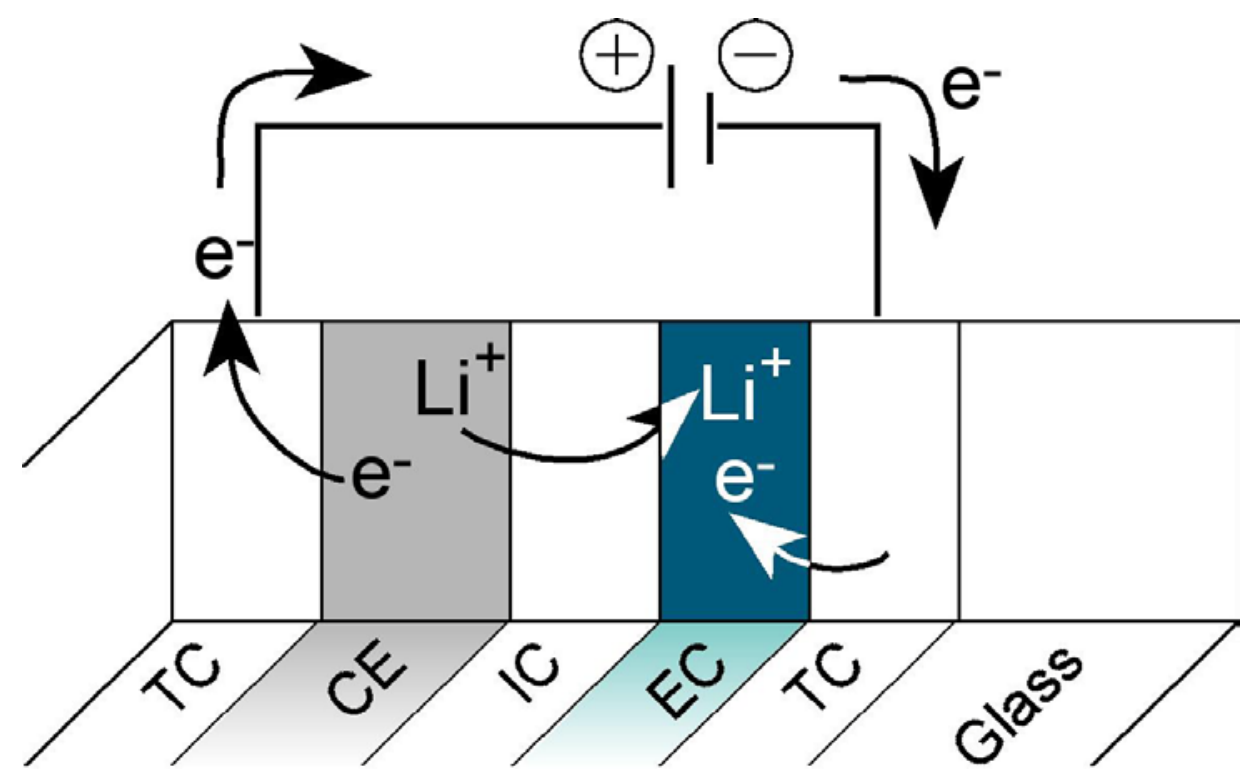

Figure 5 - The SageGlass ${ }^{\circledR}$ EC device showing the motion of the charged species around the 'circuit' as the device is colored. on the inner surface of the sheet of glass facing the outside of the building. This is standard for many glazed coated products and the convention is illustrated in Figure 4, where the relevant surface is labeled as surface 2.

An electrochromic window comprises a stack of thin films, usually deposited onto a glass substrate, configured so that a voltage can be applied to induce a transmission and/or reflection change. Figure 5 shows the general layout used in the SageGlass ${ }^{\circledR}$ product. Several books ${ }^{7,8}$ and scholarly articles are available explaining the configuration of a variety of different EC devices. The reader is referred to them for further details.

SAGE has developed a product which is based on a conventional five (functional) layer electrochromic stack, which has shown excellent performance in several durability tests conducted by external agencies. ${ }^{9}$ The key to the durability of the SAGE product is that the thin film stack is entirely inorganic. This allows the system to survive the effects of solar radiation

\footnotetext{
7 'Electrochromism and Electrochromic Devices', Monk, Mortimer and Rosseinsky, Cambridge University Press, ISBN 9780521822695 (2007)

8 'Handbook of Inorganic Electrochromic Materials', C.G.Granqvist, Elsevier, ISBN 0-444-89930-8 (1995)

${ }^{9}$ NREL testing carried out over a number of years. For details of the methods employed see http://www.nrel.gov/buildings/electrochromic_activities.html\#durability
} 
where any organic component, examples of which can be found in many other EC systems, may not be as durable.

The SAGE device is a series of thin films deposited onto a glass substrate one on top of the other to form a functional EC stack. Excluding some important optical tuning layers, which will be discussed later, the outermost layers are transparent conductors (TC ${ }^{10}$, which are used to apply a voltage to the active layers sandwiched between them. The active layers consist of an electrochromic (EC) layer, an ion conductor (IC) layer, and a counter electrode layer (CE). In order to maintain charge neutrality, electrical charge, in the form of electrons and ions, is shuttled between the CE and the EC layers, producing the clear and colored states respectively: the electrons are passed around the external circuit, while the ions are transported within the thin film stack via the IC. Insertion of charge into the EC layer will cause that layer to color to a depth which depends upon the amount of charge transferred. The effect is completely reversible, and is accomplished simply by reversing the polarity of the applied voltage.

The change in transparency is brought about by a cathodic electrochemical reaction where both ions $\left(\mathrm{M}^{+}\right)$and electrons $\left(\mathrm{e}^{-}\right)$are inserted into the electrochromic material so that the following is true:

$$
\begin{array}{ll}
\mathrm{EC}+x \mathrm{e}^{-}+x \mathrm{M}^{+} \leftrightarrow & \mathrm{M}_{x} \mathrm{EC} \\
\text { Clear } & \text { Absorbing }
\end{array}
$$

where EC represents the electrochromic material. Obviously, materials which produce more absorption for a given amount of charge transferred ${ }^{11}$ are preferred here. There are numerous reasons for this. The depth of coloration for an EC film depends on the total amount of charge inserted into the film, which in turn depends on the current flowing for a given time $(Q=I t)$. By increasing the electrochromic efficiency, the amount of charge which has to be transferred to achieve the desired coloration is reduced, and so both the switching time and power consumption are reduced.

In order for a change in optical properties to happen, the charged species must be stored somewhere within the stack when the EC device is in the clear state. This is the role of the CE layer ${ }^{12}$ otherwise known as the charge storage layer. In some cases the CE is a material which colors anodically, meaning the coloration takes place as the electrons and ions are extracted from the material. Another term for this is that the EC and CE materials color in a complimentary sense and the coloration depth for a given amount of charge transfer is increased as a result of using such an anodic CE layer.

Having two complimentary EC materials provides several advantages. First, as indicated previously, a greater depth of coloration can be achieved for a given charge transfer. Also, the window can be colored more rapidly, as each unit of charge transferred between the electrodes provided two units of absorption, one from the insertion into the EC layer and one from the extraction from the CE layer. Additionally, the spectral selectivity of the absorption can be tailored somewhat by modifying the relative amounts of each material to compensate to some extent the perceived transmitted color of the EC device.

\footnotetext{
${ }^{10}$ Transparent conductors (TC's) are optically transparent and electrically conductive when deposited in thin layers.

${ }^{11}$ The amount of absorption produced for a given charge insertion is known as the electrochromic efficiency.

${ }^{12}$ The nomenclature 'CE' stands for Counter-Electrode, and is derived from the field of electrochemistry, and is one of the electrodes in the well-known three-electrode system used for characterization of electrochemical systems.
} 
The standard EC device has the CE and EC layers separated by a material which allows the transport of the ions, but blocks the transport of the electrons. This material is therefore referred to as the ion conductor (IC) layer. This prevents the electrons flowing freely across the stack, which would lead to a situation where the device will not color due to it effectively being shorted out. Obviously, for window applications the ion conductor needs to be transparent.

This three layer arrangement is then sandwiched between two transparent conductive layers which serve to supply and remove electrons from the stack. The requirements for these layers are that they are transparent and conductive, and again have refractive indices close to those of the EC and CE layers to further minimize reflections. Furthermore, the more conductive these layers can be made, the less voltage drop will occur across ${ }^{13}$ them during switching when current is flowing, so the voltage difference applied to each individual area of the device will be as even as possible across its width, so that the transmission change, which will depend on the voltage applied to the stack, will be as uniform looking as possible. Unfortunately, making these layers more conductive by simply making them thicker has a number of drawbacks: these conductive layers tend to be made from expensive materials, and also as they become thicker they exhibit more absorption and haze ${ }^{14}$.

Further enhancements to the EC device can be made by adding index matching layers both between the glass substrate and the lower transparent conductor, and on-top of the upper transparent conductor. These are not shown in Figure 5, but are important to provide a method of controlling the reflected color, which is another important commercial consideration.

Due to the requirements that a voltage difference be supported across the layers of this device, it is important that the two transparent conductors do not 'short' together, as this would prevent the adjacent region from coloring, leading to a clearly visible defect, (known as a short). Shorts are one of the most insidious defects in this type of device, as they can be produced by any small defect present on the glass substrate which is of the order of, or larger than the thickness of the stack. This means that the production facility needs to guard against all potential defects, therefore requiring that the critical processing occurs in a clean room, and that the glass substrate need to be very clean, and therefore amenable to aggressive cleaning processes.

Deposition of the layers comprising the device can be done in many different ways, although large area sputtering is the most popular because of the economies of scale, flexibility to coat different substrates and the wide range of materials which can be deposited. In order for EC windows to achieve wide-scale market penetration, production costs need to be minimized. Adoption of large-area sputtering to the exclusion of other processes - such as sol-gel deposition or evaporation - is an important step towards this goal, but additionally cheaper materials need to be used to drive the cost down still further.

One additional deposition method which has to be considered is that of atmospheric pressure chemical vapor deposition (APCVD), where thin film materials are deposited directly on the float line over large areas. The advantage of this technique is that it is cheap, but it suffers from one or two draw-backs: There are only a handful of materials which have been successfully

\footnotetext{
${ }^{13}$ Current flow in the transparent conductors occurs largely in the plane of the substrate, prior to flowing through the device (perpendicular to the plane of the substrate).

${ }^{14}$ Haze is defined as the ratio of the diffuse (i.e. scattered) component of transmitted light to the total amount of light transmitted by a thin film for the wavelengths of light of interest, usually the visible rangeand appears to make the films milky white.
} 
deposited in this manner, and next, the float glass manufacturing process is somewhat dirty ${ }^{15}$ at the level of concern here.

A second area for improvement in EC glazing and addressed in this project, is in the technical performance. This principally means improving the dynamic range - increasing the clear state transmission or solar heat gain coefficient (SHGC) ${ }^{16}$, and decreasing the colored state transmission - without compromising the power consumption or switching time. In addition, further improvements can be made in the color of the device both in reflection and transmission.

Part of this project was aimed at technical improvements aligned with these aims, and some of the details are described here.

In order to improve the overall SHGC it is necessary to reduce the amount of absorption in some or all of the layers. Task 2 will address reduction of these absorption contributions from several of the layers. Significant progress will be shown to have been made.

At the beginning of the project, the SageGlass ${ }^{\circledR}$ device used an APCVD product as the lower transparent conductor, and so the objective of Task 2.1 was to replace this layer with an appropriate material which can be sputtered. The net effect of this is to increase the yield, thereby reducing the cost. This was the work proposed for Task 2.1.1, which will be shown to have been completed successfully.

In principle, the replacement of the lower transparent conductor appears to be simple. Unfortunately, in practice it has been found that this can lead to two distinct problems:

(1) The device properties are significantly degraded, leading to devices which draw significantly higher leakage currents, and consequently do not color as well, and

(2) The replacement transparent conductor does not survive the electrochromic coating process, leading to poor conductivity, and reduced transparency.

The purpose of Task 2.1 was to produce a material which did not suffer from those drawbacks, and to better understand the complexities responsible for the problems. It will be seen that this has been accomplished successfully.

Significant improvement in the clear state transmission can be achieved by improving the transparency (i.e. reducing the absorption) in the EC and CE layers through material improvements, and for the CE this was covered by Task 2.2. Further improvements aimed at improving the SHGC range involve careful selection of the materials and their thicknesses to produce the best optical design possible. This was the subject of Task 2.3. Significant progress in this area will be discussed later.

Task 3 was broadly associated with reducing the cost of the EC windows without compromising the performance. This involved replacement of some of the existing higher cost elements. In Task 3.2, we proposed replacement the low-sodium glass substrate with standard float glass without necessarily compromising the optical performance if at all possible. Float glass in known to have significant numbers of surface imperfections compared with LCD glass, and Task 3.2.1 was intended to address this by developing a cost-effective, beneficial and simple cleaning process. It will be seen that these elements of the project have been fully completed.

\footnotetext{
${ }^{15}$ Primarily due to tin specks, flakes from re-deposited coating material and voids.

16 The SHGC is the fraction of the sun's incident energy which is transmitted through the window.
} 
It is well established in the liquid crystal display (LCD) industry that sodium can have a deleterious effect on the properties of the transparent conductors deposited onto float glass, due to the relatively high mobility of the sodium ions in the glass substrate at elevated temperatures attained during thin film deposition. These sodium ions can diffuse into the TCO, thereby causing a decrease in the conductivity and transparency. Task 3.2.2 intends to answer the question of whether this will be an issue for the EC devices produced on such substrates.

Further possible cost reductions are associated with the production process. It has already been determined that the most cost-effective method for thin-film device production on large area glass substrates is sputtering. Sputtering has many advantages which have been discussed previously, but also one or two important limitations. Essentially, the cost of the coating (excluding the component directly attributable to the cost of the raw material itself) is directly proportional to the throughput. This means that a higher deposition rate allows the line to run at a higher line-speed ${ }^{17}$ resulting in a higher throughput. Higher deposition rates can be brought about by increasing the power applied to the cathode. The amount of power which can be applied to the cathode is limited primarily by the ability to cool the target, and by the physical robustness of the material itself. Applying too much power to a cathode can lead to catastrophic material failure, which obviously must be avoided. Many factors influence this, including the method by which the target material is bonded to the backing plate, and often the density of the target material. Task 3.3.1 will show improvements which will lead to throughput improvements in the future.

Historically, sputtering targets were flat plates of the desired material which were bonded to a cooled (usually copper) backing plate ${ }^{18}$. The disadvantages of this configuration are that: the erosion track is limited to a small area of the target, 'the racetrack', and so the amount of material remaining after the target is burned through is substantial, leading to low target material utilization and hence wasted material; in some situations, material can be re-deposited onto the areas of the target away from the racetrack, and particularly in reactive sputtering processes, these can become electrically charged, thereby leading to possible arc events ${ }^{19}$ which can damage the coated material. A well known solution to the problems associated with a planar magnetron is to use a rotatable magnetron ${ }^{20}$ which overcomes these limitations.

In the rotatable cathode, a cylinder of the material is rotated through the stationary magnetic field, thereby preventing the build-up of potentially insulating layers on the cathode, and utilizing significantly more of the target material. It is this technology which is now most widely used in high throughput sputtering machines. One slight complication, particularly for mixtures of materials is that now the target must be fabricated as a cylinder. It is not so easy to form and bond material in cylindrical shapes as it is for the case of flat plates. A solution to this is to plasma spray liquid metals onto the cylinder, although, naturally, this is not without further complications, particularly when the materials have widely differing physical properties, as this can lead to difficulties of composition control.

\footnotetext{
${ }^{17}$ Line-speed is the rate that the glass is moved past the sputtering cathode, and is measured in units of velocity (i.e. $\left.\mathrm{ms}^{-1}\right)$.

${ }^{18}$ Referred to as a planar magnetron sputtering cathode.

${ }^{19}$ An event where a build-up of charge produces an electric field capable of spontaneously discharging to an area of lower potential, in a manner similar to a miniature lightning strike.

${ }^{20}$ See for example patents US4356073 (McKelvey) and US5213672 (Hartig et al) for details.
} 
A second component of higher throughput is the fraction of time that the coating equipment is available for production (otherwise referred to as uptime). One of the limiting factors for the SageGlass ${ }^{\circledR}$ process is that several of the targets need routine maintenance for cleaning. One further aim of this Task is to develop target materials which give longer maintenance intervals.

Increasing the yield is also an effective way of reducing the overall cost of the product. One way to do this is to automate some of the functionality required to control the coating process. Currently, the coater operator interprets measurements of optical and thermal measurements, and adjusts the controls of the coater accordingly to obtain the desired thin-film properties. It is completely feasible to automate this process to remove the possibility of human error, and increase the responsiveness of the feedback between measurement and implementation. Task 3.4 will address this. Further instrumentation will be developed, and significant effort expended on understanding the process so that appropriate feedback can be automatically applied with the aim of improving the repeatability of the coating process.

The preceding section is intended to place the following in context so that the reader not intimately familiar with the project and technology involved will be able to understand, and have a reference for some of the terminology used. 


\section{Discussion of Results by Task}

\section{Task 2: Improve SHGC Range from 0.09 to 0.53 or Higher:}

The objective of this task was to improve the SHGC range - the difference between the SHGC in the clear state and in the dark state (given in Table 1) - predominantly by increasing the clear state transmission. This was to be done by reducing the absorption of the films in the stack, as well as replacing the glass substrate.

\begin{tabular}{|l|c|c|c|}
\hline $\begin{array}{l}\text { Tint } \\
\text { State }\end{array}$ & \multicolumn{1}{c}{$\begin{array}{c}\text { Visible } \\
\text { Transmittance }\end{array}$} & U-Factor* & SHGC \\
\hline Clear & $62 \%$ & 0.28 & 0.48 \\
\hline Tinted & $3.5 \%$ & 0.28 & 0.09 \\
\hline
\end{tabular}

Table 1. SAGE EC performance specifications at the beginning of the project. ${ }^{*} \mathrm{BTU} / \mathrm{hr} / \mathrm{ft}^{2}{ }^{\circ} \mathrm{F}$
The areas addressed were to be the lower transparent conductor, the counter electrode (CE) and the substrate. Success would allow more design flexibility by removing the necessity to use the APCVD coated float glass, allowing alternative glass substrates to be used, and expand the choice of materials which can potentially be used in the device's structure, with the net result that the visible transmission of the device will be increased, thereby leading to an increase in the SHGC of the clear state.

Based on preliminary evaluations, both the APCVD transparent conductor and CE films have some absorption in the visible range which result in a slight attenuation of transmission in the clear state. Reducing this absorption will increase transmission and SHGC as will the use of less absorbing glass. When all of the above enhancements are coupled with better design of the optical stack to reduce reflections, the anticipated SHGC should be achievable.

The task was therefore split into three subtasks: the first dealing with the replacement of the lower transparent conductor (Task 2.1), the second concerned with the replacement of the CE (Task 2.2), and the third aimed at improving the design of the optical tuning layers (Task 2.3).

\section{Task 2.1: Replace the Bottom Transparent Conductor $\left(\mathrm{TC}_{\mathrm{b}}\right)$.}

At the beginning of the project, the only substrate of practical use was the commercially available APCVD coated float glass, which was only offered in one convenient thickness of $6 \mathrm{~mm}$ at a sheet resistance of around $12-15 \Omega / \mathrm{sq}$. Previous attempts to replace the lower transparent conductor had resulted in devices which showed degraded performance as a result of higher leakage current. This included devices where the APCVD TCO was over-coated with additional conducting material. The mechanism for this was not clear, but several theories were under consideration, including surface roughness, possible defects, and effect of the different substrate material on subsequent film deposition.

The APCVD coated float glass shows considerable absorption, which is undesirable for increasing the clear state SHGC as shown in Figure 6. The absorption is derived from two sources; the float glass substrate itself, and the conductive coating. Elimination of both sources of absorption will be beneficial, and the only practical way to do this is to develop a different transparent conductor which works in the context of the EC device, thereby removing the need for float glass, so that a less absorbing glass substrate can be used, along with a more transparent coating. 
Reductions in the absorption of the lower transparent conductor $\left(\mathrm{TC}_{\mathrm{b}}\right)$ can have a significant impact on the overall amount of transmitted light, and hence help improve the SHGC range as desired. In order for this to be successfully implemented as part of this project, however, it is necessary to retain low sheet resistance to prevent problems with switching the EC device as already discussed.

\section{Progress Made During this Project}

Early in the project, optical measurements of the existing APCVD TCO were made and these are summarized in Figure 6 and compared with some commercially available ITO ${ }^{21}$ and a novel TCO deposited in our production coater. While the novel TCO is not as transparent as the commercially available material, it was found that when devices were fabricated on the commercial ITO, the functionality was significantly degraded, leading to EC devices which exhibited high leakage current and very limited dynamic range. The novel TCO did not display these drawbacks. It was not clear why the devices deposited on the commercially available ITO behaved so poorly, but it was speculated that the active layers of the device somehow interacted

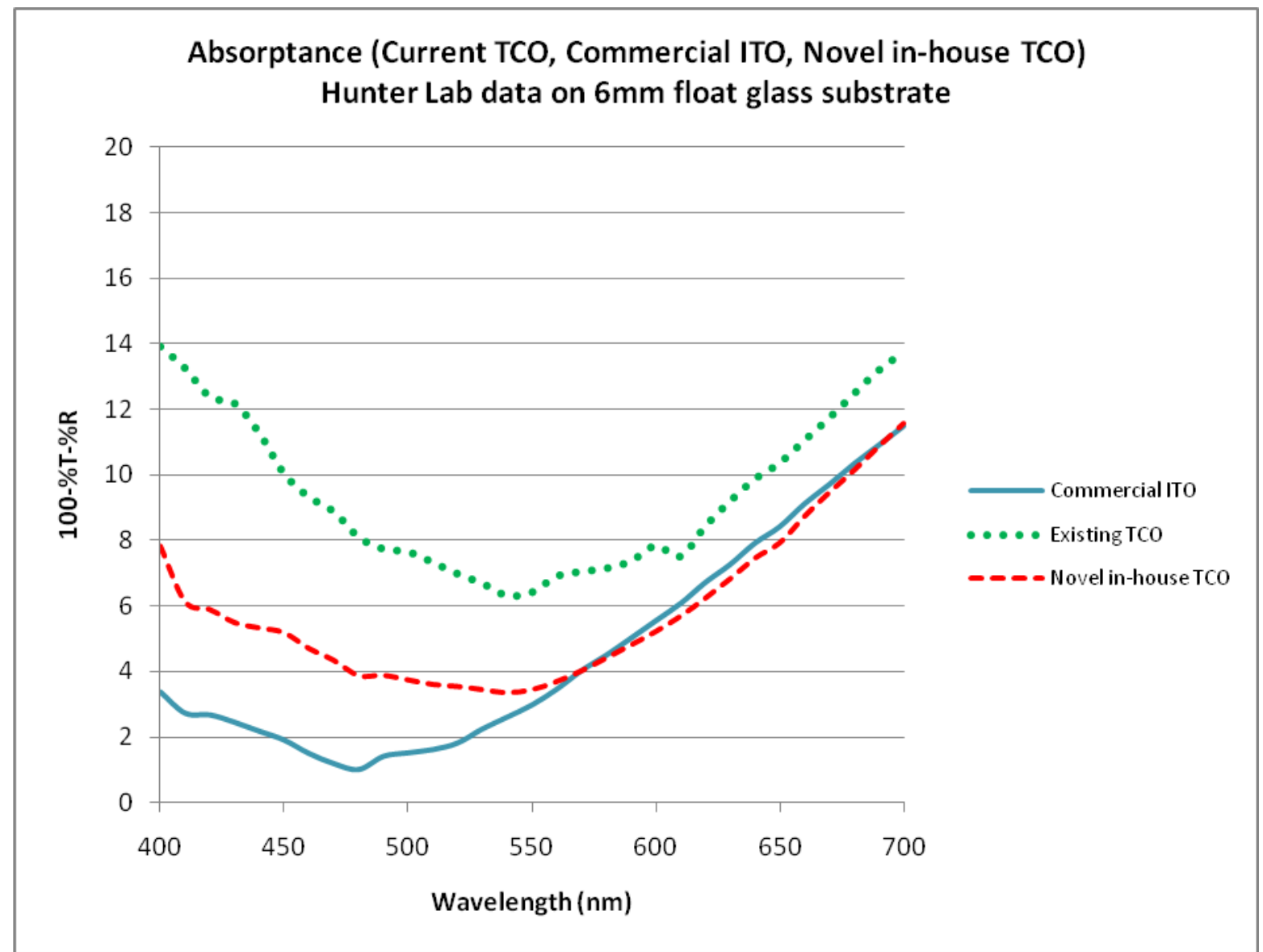

Figure 6 - Absorption, calculated as (100 -Transmission - Reflection) for the TCO systems discussed in the text.

${ }^{21}$ ITO is Indium Tim Oxide, the most commonly used and widely available transparent conductive material, used in almost all LCD displays manufactured today. 
with the TCO, leading to the poor performance.

Although the early attempts to develop novel in-house TCO resulted in films which were not as transparent as the commercially available TCO, they still showed significant improvements over the existing APCVD TCO in terms of the optical properties of the layer itself. Subsequent work on this task was therefore aimed at understanding the requirements for the layer to enable even better performance to be achieved, as well as optimizing the deposition of EC devices on this material, as there is a clear dependence on the behavior of the device on the substrate.

A series of transparent conductive oxide (TCO) trials were therefore performed to understand the role of deposition power and gas flow rate on the film properties. Extending the deposition time of our current TCO process led to decreases in sheet resistance which were roughly inversely linear with deposition time - or in other words thickness - along with a corresponding increase in haze.

The other deposition parameters which are known to influence the optical and electrical properties of transparent conducting films are the deposition power, substrate temperature and the sputter gas composition. Several experiments were carried out to understand the effects of these parameters on both the TCO behavior - primarily transparency and conductivity - as well as the electrochromic performance. Significant progress has been made in this area, enabling devices with much better electrochromic performance and lower absorptivity to be fabricated.

\section{Device Performance on Alternative Substrates - Extrinsic Properties}

Several experiments were aimed at understanding the differences seen in overall device performance when different transparent conductive oxide (TCO) materials are used. It is not intuitively obvious why there should be a significant difference, but we have found this to be the case.

The experiments carried out have concentrated on running designed experiments (DOE's) where the variable factors include the transparent conductor material as well as other selected process parameters such as film thickness and thermal processing parameters.

For example, we have found that the electronic leakage current - defined here as the current flowing when the device is fully colored or fully bleached - depends on the TCO material. Figure 7 shows the impact of the TCO material on the measured leakage current for a range of processing conditions (shown as the different colored points on the plot, with higher levels shown colored in red, and lower levels blue). This chart shows the leakage current for five different TCO materials (here labeled as 'Substrate'). It can be seen that the lowest leakage current seen for each different substrate is a function of the TCO material, illustrating the differences we are concerned with here. 


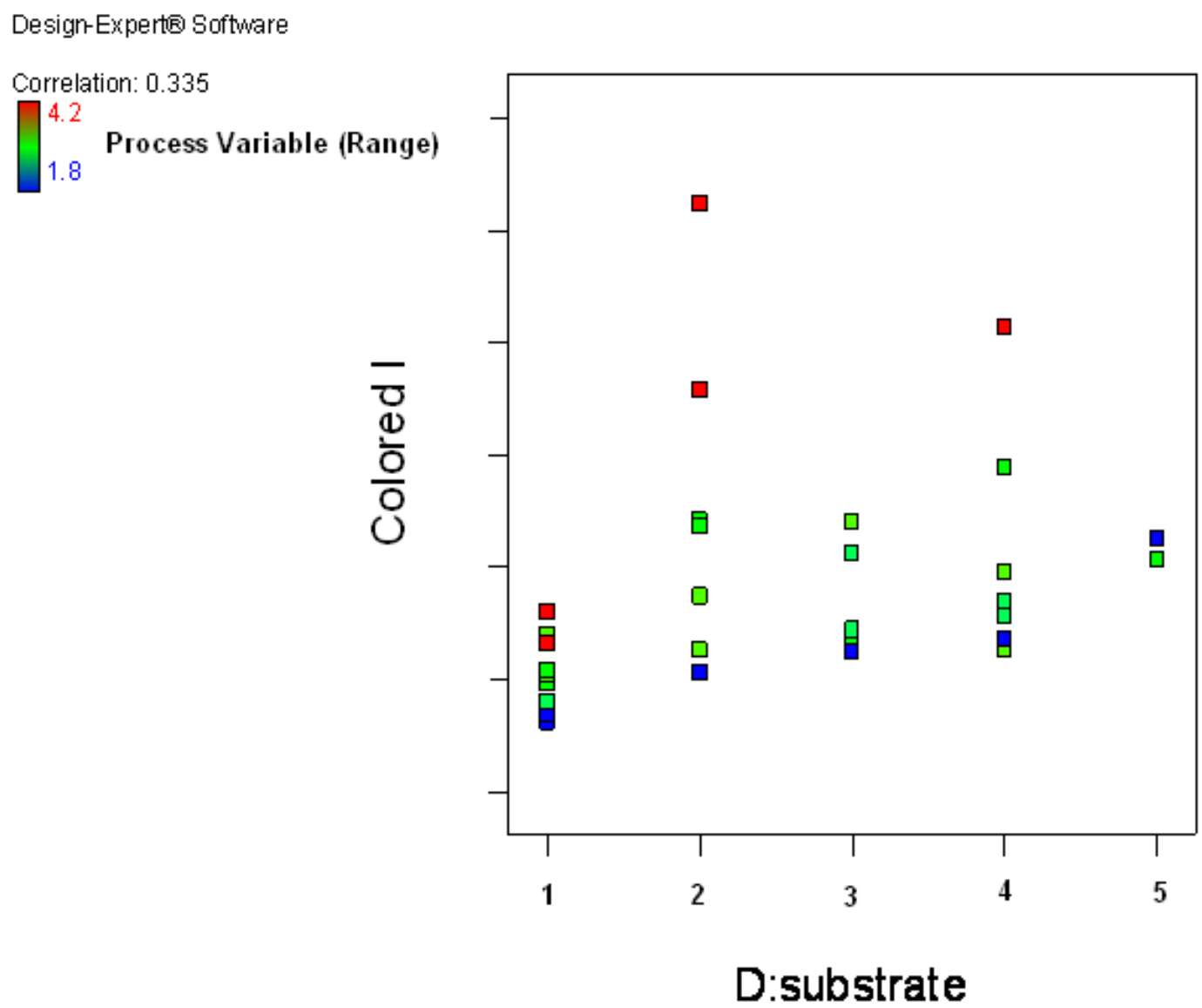

Figure 7 - Measured leakage current from an experiment carried out on a variety of different transparent conductor substrates, illustrating the variation as a function of the TCO.

Leakage current is important because for larger devices, when the leakage current is flowing, there is more voltage dropped across the series resistances - i.e. the transparent conductors leaving less to be dropped across the electrochromic stack, leading to less coloration, and often, due to the geometry of the device, non-uniform coloration. It is this problem which makes this seemingly simple task more complicated.

So the problem was to understand the relevant process factors to ensure that the device performance was not degraded as a result of replacing the existing TCO with one of better optical properties.

As a result of a series of careful and painstaking DOEs, progress has been made in understanding the role of the Ion Conductor (IC) in the all sputtered stack, and this has improved the performance of the devices on alternative substrates. Further DOEs designed to understand the contributions made to the device behavior with different device processing parameters have been done, and the resulting incremental improvements have been incorporated in the process. In some cases this has required some fairly major modifications to the process equipment, such as rearranging the cathodes, and installing new heaters, and modifying power supply properties. 
In order to understand the differences seen between films deposited on different substrate material, we carried out an extensive electrochemical investigation. Initially, this has involved a study of the electrochemical potentials associated with the different depositions with a view to relating these to the electronic properties of the systems. First, we compared open circuit potentials (OCP) of samples deposited on different substrates with respect to a reference electrode in a liquid electrolyte. There was a noticeable discrepancy between the values measured on the different substrates.

In an attempt to modify the relevant interface, films were deposited with different sputtering conditions. These showed significant changes in open circuit potential indicating a significant modification of this interface. It is anticipated that with some further work beyond the scope of this project, this can be used to provide a valuable process control tool.

There was also a renewed effort to determine whether the differences seen in device performance are as a result of intrinsic differences between the film stacks, i.e. electronic properties of the layers themselves, or extrinsic defects due to differences in levels of microscopic contamination at the interfaces between the various active layers. In order to eliminate this issue, significant changes were made to the order of the cathodes within the Production Coater. Rearranging the order of the cathodes allowed deposition of the critical layers sequentially passing through areas where contamination may occur. The benefits of reducing this contamination are that the interfaces between the lower transparent conductor and the electrochromic layer, as well as the electrochromic layer and the ion conductor are protected, which could lead to reduction in the leakage current.

Experiments were performed to determine the variation of leakage current with processing sequence - devices were constructed with different cathode arrangements and propensities for contamination.

No significant difference could be determined, strongly suggesting that at least for the conditions used in this experiment, the leakage current was not being determined by extrinsic factors.

\section{Device Performance on Alternative Substrates - Intrinsic Film Properties}

Considering now the intrinsic film properties, we examined the electrochromic film stack using cross-section transmission electron microscopy. Samples were prepared using a focused ion beam; this produced a very large area that was thin enough for electron energy loss spectroscopy and atomic resolution of the respective thin films and interfaces. We found that modifications to the bottom TCO have a significant impact on the degree of crystallization of the EC (cathodic) electrode layer. A correlation of the observed microstructural changes with diffusion constants measured using electrochemical methods was attempted, and it appeared as though there was a significant difference between the diffusion constant for a film deposited on the different substrates. It is not clear how this affects the leakage current, but it may have an impact on the switching speed of the EC device.

\section{Summary of Progress compared to Goals}

We were able to deposit some extremely good lower transparent conductor material with very high transparency and good sheet resistance. This led to deposition of 18” x 18” devices with properties which satisfy one of the major milestones of the project, showing excellent dynamic range and switching kinetics. 
Further progress has been made in this area, and this has been the subject of considerable focus during this reporting period. Devices have been deposited with a wide range of lower transparent conductor processing conditions, and this has allowed us to better understand the relationship of the electrochromic properties with these processing conditions.

Studies to investigate the effect of deposition parameters on the properties of the transparent conductor have yielded excellent results. These include, but are not limited to studies of sputter gas composition, target composition, power supply configuration, pressure and power. The transparent conductor films have been characterized in terms of the transparency and conductivity, and these properties -amongst several others. The net result is that we are converging towards a solution which will provide high conductivity and high transparency, which is compatible with the anticipated production requirements.

\section{Task 2.1.1: Use Higher Transmittance Glass}

Replacement of the bottom transparent conductor as described in the previous task presents the opportunity to replace the base glass with something less absorbing than standard float glass, thereby resulting in an increase in the visible transmission. Furthermore, it may be important to use a glass substrate with a low coefficient of thermal expansion (CTE), as this can minimize early production risks associated with the glass expanding and breaking when heated. The objective of this task is therefore to do just that, replace the glass used for the SageGlass process at the start of the project with something which has a higher transmission and has a low CTE.

Corning Eagle XG glass ${ }^{22}$ has a visible transmission of $92 \%$ compared with $87 \%$ for soda-limesilica float glass, and also has a much lower CTE than float glass ${ }^{23}$.

Also, it is important that the device functionality remains unaffected by such a change and that the overall optical improvement justifies the probable increase in cost as a result of using such a specialty glass.

\section{Progress Made During this Project}

Several experiments with glass produced using a method other than the float process ${ }^{24}$ have been carried out in order to test the feasibility of using such glass for routine experiments. Functional devices were produced, in that the dynamic range was wide enough for an acceptable product, but there are several drawbacks to using this approach routinely.

This glass can generally only be obtained in thicknesses which are at most of the order of 1$2 \mathrm{~mm}$. This is not thick enough to allow the glass to be tempered, and so in order to prevent breakage within the coater, the edges must be prepared in such a way as to minimize any microfractures. This preparation results in extremely sharp corners, as the usual method of eliminating the sharp edges by edge grinding introduces significant edge damage and microfractures. These sharp corners can cause significant abrasion of some of the mechanical elements as the glass

\footnotetext{
${ }^{22}$ This is available from Corning. For more information see http://www.corning.com/displaytechnologies/en/products/eaglexg/index.aspx

${ }^{23}$ http://www.pgo-online.com/intl/katalog/list_sheetflat_glass.html gives the CTE for Eagle Glass as $32 \times 10^{-7} /{ }^{\circ} \mathrm{C}$ compared with $87 \times 10^{-7} /{ }^{\circ} \mathrm{C}$ for float glass.

${ }^{24}$ Float glass is a sheet of glass made by floating molten glass on a bed of molten metal, typically tin. This method gives the sheet uniform thickness and very flat surfaces. Modern windows are made from float glass. Most float glass is soda-lime glass.
} 
passes through the process, such as carrier stops, roller wheels, etc., leading to significant numbers of small abraded particulates. The contamination takes the form of small particles of a variety of materials, and these can then get back into the production process causing significant yield loss due to generation of shorting defects.

Also, some of the cleaning processes are set up for 6mm thick glass, which is the standard for the present process. This means that the washing processes are compromised, and it is disadvantageous to adjust the washers to accommodate the thin glass, as it is likely to cause abrasion on the precision components of the washers, etc. due to the sharp edges of the glass as discussed earlier. The net result is that the thinner glass is not cleaned as effectively as the $6 \mathrm{~mm}$ thick glass, leading to significant problems in experimental interpretation, because the defects induced as a result of the contamination prevent areas of the devices from coloring fully, thereby introducing the possibility of misinterpretation of the experimental results.

Most challenging, however, was the problem of adjusting the various heating steps to accommodate the lower thermal mass of the substrate. This was really a matter of running a significant number of experiments to adjust the heating processes at the appropriate stage of process development. In order to make this as efficient as possible, the process was carried out only when the defect problems had been addressed.

A significant effort was expended in order to understand the contribution of the substrate to the level of defects seen in the finished devices. This involved deposition of standard devices on a variety of thin (of the order of 2-3mm) glass substrates including float glass, and a more expensive composition of the type used in the LCD industry. Several different substrate preparation techniques were examined with a view to understanding the impact of substrate defects derived from the float glass itself. Each defect seen on the resulting devices was catalogued and characterized, and it was found that there was very little contribution to the overall defect level which could be directly attributed to any defects deriving directly from the substrate, even from the float glass substrates which were assumed to suffer from more defects such as tin specks and stones ${ }^{25}$ from the float process itself.

A further separate evaluation was made using lower iron containing float glass, whereby devices were made on this lower iron glass. Control samples deposited concurrently with these devices were made on standard float glass. The results indicated that there was little difference in the transmitted color of the clear state, but the overall level of transmission in the clear state was increased by between 1 to $2 \%$. The dark state remained unchanged.

The reflected color for the clear state was shifted slightly, but it is anticipated that this can be correctly simply by adjusting the optical design of some of the elements in the stack.

\section{Summary}

The progress made confirms that there is no fundamental impediment to the integration of a different glass substrate - particularly a high quality LCD type glass - into the SageGlass EC process. Improvements in the overall transparency in the clear state have been achieved with no detrimental effect on the dark state.

\footnotetext{
${ }^{25}$ The use of the word "stone" is usually meant to refer to defects generated from the internal furnace wall of the float bath known as refractory brick. As the molten glass pool is processed, the molten glass erodes the inner brick wall causing small pieces of the brick to become suspended in the molten glass solution. After the glass product is formed, these "stones" are embedded in the final product.
} 


\section{Task 2.2: Replace Existing CE Material}

The objective of this task is to make a modification to the CE material which will result in a less absorbing layer, again leading to a higher visible transmission. This was to be achieved by using a material with higher electrochromic efficiency ${ }^{26}$ than the current material as shown in Figure 8 allowing the same dynamic range to be

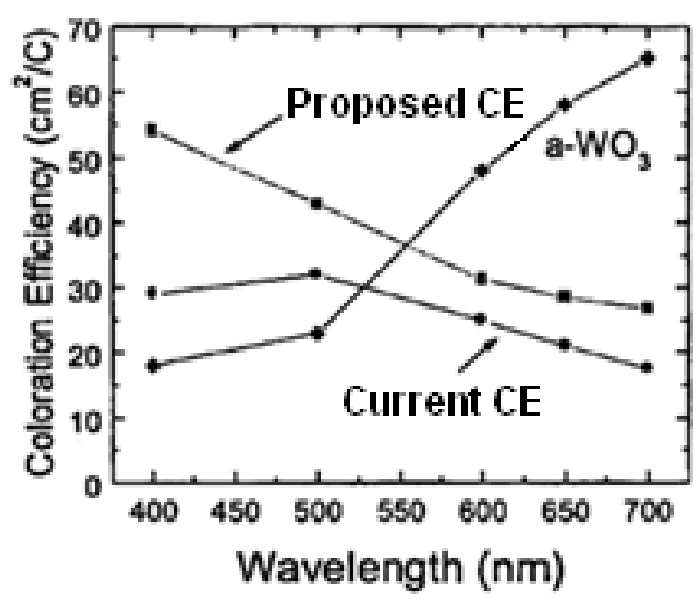

Figure 8 - Coloration efficiency (amount of absorption produced per unit of charge inserted/extracted) for some candidate materials. obtained with a thinner and therefore more transparent CE layer.

\section{Test Cell for Evaluating New Counter Electrode Samples}

In order to achieve this objective, an important task was to set up the testing equipment to evaluate the optical and electrochromic performance of new candidate materials for the counter electrode. Accordingly, we designed and fabricated a low cost, prototype test cell that combines a light emitting diode (LED) light source, a light meter, and an electrochemical cell filled with liquid electrolyte, all contained under dry, inert gas. This is shown in Figure 9.

Several of these test cells allowed us to electrochemically switch a large number of counter electrode samples between clear and dark states at the same time. Meanwhile, the associated electronic hardware recorded data to determine the amount of electrical charge supplied to thin film sample to obtain a corresponding change in light transmission. We also determined the rate of change and the repeatability of the change.

By statistically comparing the results for a wide variety of samples, we were then in the best possible position to select the best formula for modifying the counter electrode.

Literature data suggested that these proposed materials exhibit reduced absorption in the blue wavelength range which could potentially yield a more neutral tint when combined with the predominantly blue absorption of the electrochromic layer.

${ }^{26}$ Lee, Se-Hee. Electrochromic Counter Electrode. US 6859297 USA, February 22, 2005. 


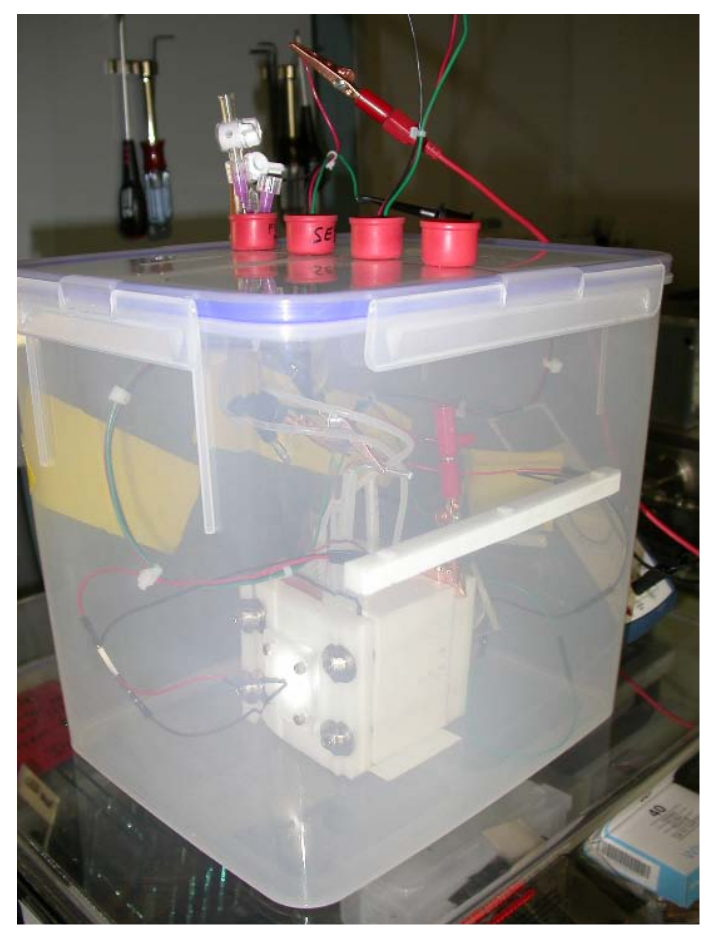

Figure 9 - Low cost test cell for counter electrode samples. A sample is clamped onto the end of a hollow Teflon block to form both the window and electrode of an electrochemical cell. Light from a light emitting diode (LED) shines through the sample onto a light meter. The entire cell is contained under dry, inert gas.
Composite targets of each material were fabricated from plates and strips of the materials. The strips lay across the plate reducing in number across the length of the plate producing a composite target with a gradient in composition. Films will be intercalated in an electrochemical cell and wavelength dependent transmission will be monitored using an Ocean Optics spectrometer $^{27}$.

Understanding the details of the properties of the present CE material system was important for the successful replacement of this layer with a more transparent material, as the new material needs to perform as well if not better than the current material.

In order to do this, electrochemical impedance spectroscopy experiments to analyze CE samples were carried out. Ion transport experiments were carried out in CE half cell samples.

Several approaches to depositing the multielement counter electrode (CE) thin film layer were explored, including co-sputtering and sequential sputtering. The $\mathrm{CE}$ thin films produced were evaluated using half cell electrochemical analysis techniques, and optical properties were measured in-situ.

One of the test compositions showed significantly improved performance when compared to our present material. The switching time of the new experimental single CE film was reduced by $45 \%$ and the dynamic range was increased by $29 \%$. However, the electrochromic efficiency was not increased as anticipated. Also, it is not clear whether these improvements would translate into improved device performance.

\section{Task 2.3: Optimize Thin Film Stack}

In line with the overall aim of this task which was to increase the clear state transmission to obtain a wider range of SHGC, it was proposed to improve the transmission by optimizing the optical properties - thickness and refractive index - of each of the layers in the thin film stack. A significant increase in transmission can be achieved in this way. Following successful replacement of the lower TC as discussed above, standard anti-reflection (AR) thin-film coatings can be deposited between the glass substrate and the EC layers as well as over the top transparent

\footnotetext{
${ }^{27}$ Ocean Optics is a company specializing in miniature spectrophotometers. For more details www.oceanoptics.com
} 
conductor (TC). Also, if the refractive indices of the EC layers are better matched together, this will greatly reduce internal reflections leading to higher transmission.
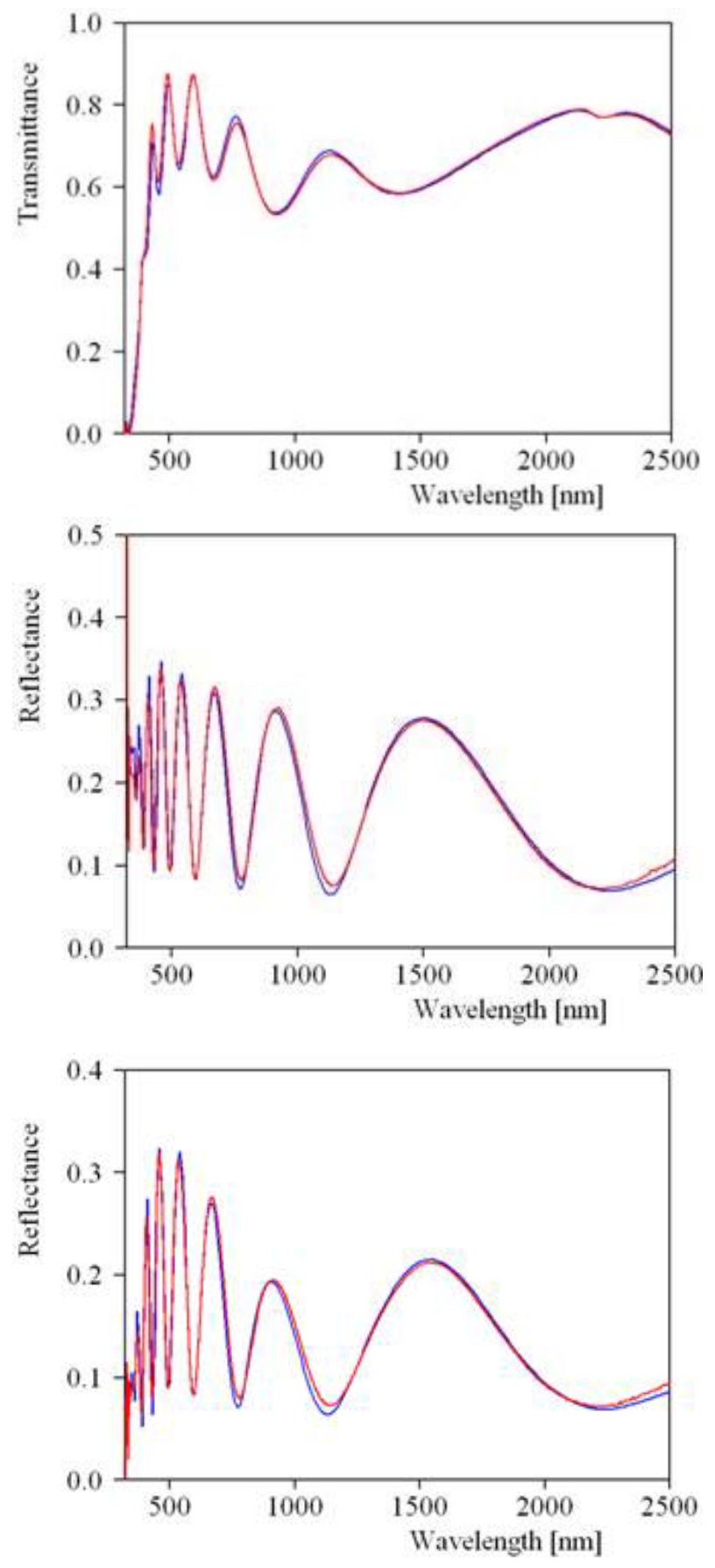

Figure 10 - example of the quality of the optical measurement and modeling of a single layer deposited in this work. (Red=measurement, blue=Model)
The deliverable for this task was a prototype EC window with a higher SHGC in the clear state than was being achieved at the beginning of the project, whilst retaining the value of 0.09 in the tinted state. This is clearly in line with the overall project objective. Each subtask will result in different device configurations with, first, $\mathrm{TC}_{1}$ replaced, next, a device using higher transmission glass, then one using a more transparent CE material, and, finally, one incorporating index matching layers. The ultimate prototypical device will have a SHGC $>0.53$, and we will produce a significant number for durability testing in Task 5.

\section{Progress Made During the Project}

Prior to full optimization of the antireflective (AR) layers deposited beneath the lower TCO, it is necessary to produce a fully functional and optimized EC device. It has been determined that the AR layers deposited between the substrate and the lower transparent conductor, as well as on-top of the top transparent conductor, will both probably consist of two layers - a high index material, followed by a low index material. In order to ensure that any development of the device does not suffer from any unexpected adhesion problems as a result of leaving these layers out, it was decided to use a 'placeholder' combination, which can be optimized at a later date, for the AR deposited onto the substrate. Two suitable materials were chosen which were already available within the coater. Estimates of deposition conditions were made to obtain layers of approximate quarter wave thickness, and these have been used routinely for all the development work. No problems with adhesion were seen. 
The next task was to deposit single films of all potential materials for the AR layer and determine the refractive indices to allow for a comprehensive optical model to be constructed. This involved experimentation with different substrates as some of the low index materials did not give a good enough optical contrast when deposited on glass.

Measurement of the optical properties of the individual layers comprising the stack has been completed. The aim of this work was to arrive at a representative optical model of the entire electrochromic device, even as the device transmission is varied electrochromically. This optical characterization has been carried out by depositing the individual electrochromic layers, and then using sputtering to produce electrochromic coloration to different degrees. The optical properties were then measured and the results fitted to an optical model. The model allowed design of the optical tuning layers (undercoat layers) to be carried out efficiently, as there are requirements for the reflected color at both the clear and tinted states. These requirements are very stringent, including the absolute value of reflected color and the change in this color as the level of tint in the device is changed.

Excellent progress has been made on this part of the project, with the various parts of the model being refined prior to assembly of the whole system. Figure 10 shows the quality of the fitting for one of the layers, showing the measurement and modeled data for the transmission, and reflection from both sides of the glass.

Another important element of the lower transparent conductor project is the sequence of layers deposited beneath the conductive layer in order to modify the reflected color. These layers are referred to as the undercoat or the antireflective layers, although this latter description is not completely accurate and 'color suppression layers' is probably a more accurate description. The requirements for this layer are somewhat complicated, but can be summarized as follows:

- $\quad$ These layers - in combination with the other layers of the stack - should provide a pleasing reflected color (when viewed from the glass side) in the clear state;

- $\quad$ This reflected color in the clear state should become more neutral as the viewing angle moves away from normal incidence;

- Ideally, the reflected color should remain roughly constant as the device changes state from clear to tinted. This is somewhat complicated, as in the clear state, the reflected color is the superposition of reflections from each of the interfaces of the device.

In order to determine the required properties of the undercoat films, we have developed a method of depositing thin film gradients onto large square substrates. This allows two different films to be varied across two directions thereby providing an extremely high throughput experimental method.

Several experiments have been carried out using this technique. In order to characterize the reflection of the device in the clear state, all the films were deposited. On the other hand, to characterize the dark state, it is necessary to include all the layers up to but not including the EC layer, but with the reflection from the TCO/Air interface modified to more accurately reproduce the behavior when the EC layer is in the absorbing state. Analysis of these experiments has provided enough information to produce prototype devices. 


\section{Summary of Progress made in Task 2}

Table 1 gave some of the relevant parameters of the production devices measured at the start of the project. Table 2 shows the same parameters for a prototype device made with the thin film structure developed during this project.

\begin{tabular}{|c|c|c|c|}
\hline \multicolumn{1}{|c}{$\begin{array}{c}\text { Tint } \\
\text { State }\end{array}$} & \multicolumn{1}{c}{$\begin{array}{c}\text { Visible } \\
\text { Transmittance }\end{array}$} & \multicolumn{1}{c|}{ U-Factor* } & SHGC \\
\hline Clear & $69 \%$ & 0.33 & 0.47 \\
\hline Tinted & $2.1 \%$ & 0.33 & 0.08 \\
\hline
\end{tabular}

Table 2. SAGE Glass performance of prototype device. *BTU/hr/ft2/F
By comparison of these values with those given in Table 1, it can be seen that the range of visible transmission has been improved, both by increasing the clear state transmission, and reducing the tinted state transmission.

However, comparison of the values of SHGC

for the clear state does not show the anticipated improvement which we had hoped for at the start of the project, and suggested by the improvements made to the clear state visible transmission. The reasons for this are associated with other changes which have also been made to the stack, which are necessary for wide-scale market penetration of the product, but which are not necessarily directly part of the current project. This has resulted in the comparison shown between the start of the project and the prototype sample measured here, as not being completely valid. In order to satisfy the requirements to switch wider samples, we have made the transparent conductors more conductive by a significant amount. This has resulted in a higher reflection in the near infra-red, where previously there was some significant transmission of energy. This has probably led to the lack of improvement in SHGC observed here, but had the comparison been done with similar TC sheet resistance to the original device, then it is likely that we would have seen the improvement as predicted. Finally, it should be pointed out that the SHGC in the colored state has been reduced, which is therefore improved.

\section{Task 3: Reduced Cost High Performance EC Windows}

\section{Objective}

The objective of this task is to achieve lower production costs for the SageGlass EC device by investigating the use of lower cost materials. Obviously, it is important for the device functionality to remain unaffected by these changes. These were to replace some of the higher cost elements of the device, such as the transparent conductors, and the LCD quality glass.

As a result of replacing the high quality substrate with float glass, it was necessary to ensure that this did not compromise the low defect levels obtained with the high quality glass. This involved developing a washing process as well as understanding whether there was a possibility of the relatively mobile sodium ions from the float glass substrate having an impact on the device performance.

In addition, some process improvements will be investigated with a view to reducing costs further. This included improvements to the target materials, increasing the sputter rates, and better process controls.

\section{Task 3.1: Replace Lower Transparent Conductor}

In Task 2.1, we saw that the commercially available APCVD deposited TCO could be replaced by a novel in-house deposited transparent conductor. This involved noteworthy effort in 
understanding the impact on the device properties when the layer was replaced, as has been previously noted, it was found to have a significant effect on performance. This resulted in a device which showed excellent behavior, but has a somewhat costly transparent conductor.

The aim of Task 3.1 was to replace this costly material with one which is less expensive, but still produces a fully functional EC device. The learning from the work done in Task 2.1 can be applied to permit the functionality to be maintained, as it is generally applicable to most of the materials of this type.

The lower coat transparent conductors are generally of either lower conductivity or higher absorption, or both. As a result, it is necessary to deposit thicker layers to achieve the same sheet resistance, and this could result in low transparency or even increased haze. It is important to understand whether these possible compromises in thin film properties are significant in terms of overall device performance.

Thin films of an alternative material satisfying the criterion of being cheaper were obtained from an external vendor, and evaluated. Several experiments aimed at understanding these properties as a function of the usual film deposition parameters were carried out, but as there was no room to install a target of this material in the production coater, these films were made by several different outside organizations.

The single film results showed the expected behavior where the conductivity and optical properties behaved in a similar manner to other transparent conducting films. However, the absorption and haze for these particular films was somewhat higher than those seen for the novel TCO discussed in Task 2.1. In addition, there was some evidence that the sheet resistance could be degraded during subsequent device processing, but there appears to be a processing path which can avoid this particular issue.

This work resulted in functional devices, even without a large degree of process optimization. It is clear that this material can offer a viable alternative to the higher cost materials currently being used, and it is likely that further development will be carried out at a later date.

\section{Task 3.2: Replace Thin Low-CTE Glass with Float Glass}

We saw in Task 2.1 that it was possible to replace the lower APCVD transparent conductor with a material deposited onto a low coefficient of thermal expansion (CTE) glass substrate - of the type used for LCD displays. This glass is very high quality, and as a result can be somewhat expensive. We demonstrated that we could use this glass to produce fully functional devices but in order to reduce the cost of the device as much as possible, it is advantageous to use float glass for the substrate.

This section discusses the work carried out in order to achieve this. This includes understanding the impact of the defects associated with the float process, and then eliminating them via an aggressive cleaning process if appropriate. Also, it is important to understand whether the introduction of a material containing a high concentration of relatively mobile sodium ions will have a detrimental impact on device characteristics, particularly the properties of the transparent conductor.

We start with a discussion of the cleaning requirements and processing, and conclude with experiments and measurements carried out to understand the impact of sodium ions present in the float glass. 


\section{Task 3.2.1: Develop an Effective Cleaning Process for Float Glass:}

Float glass debris includes tin specks $^{28}$, organic contamination and dirt. The aim of this task is to produce clean substrates with a minimum of defects in a cost effective manner.

In order to characterize the cleanliness of the glass prior to EC device deposition, representative samples are measured with a $\mathrm{Dr}$ Schenk particle monitoring system $^{29}$ as shown in Figure 11, which can give a measurement of the number and size of any particles on the substrate, as well as sometimes helping to identify the type of particle. These camera systems are capable of detecting defects of size $>10 \mu \mathrm{m}$, scratches,

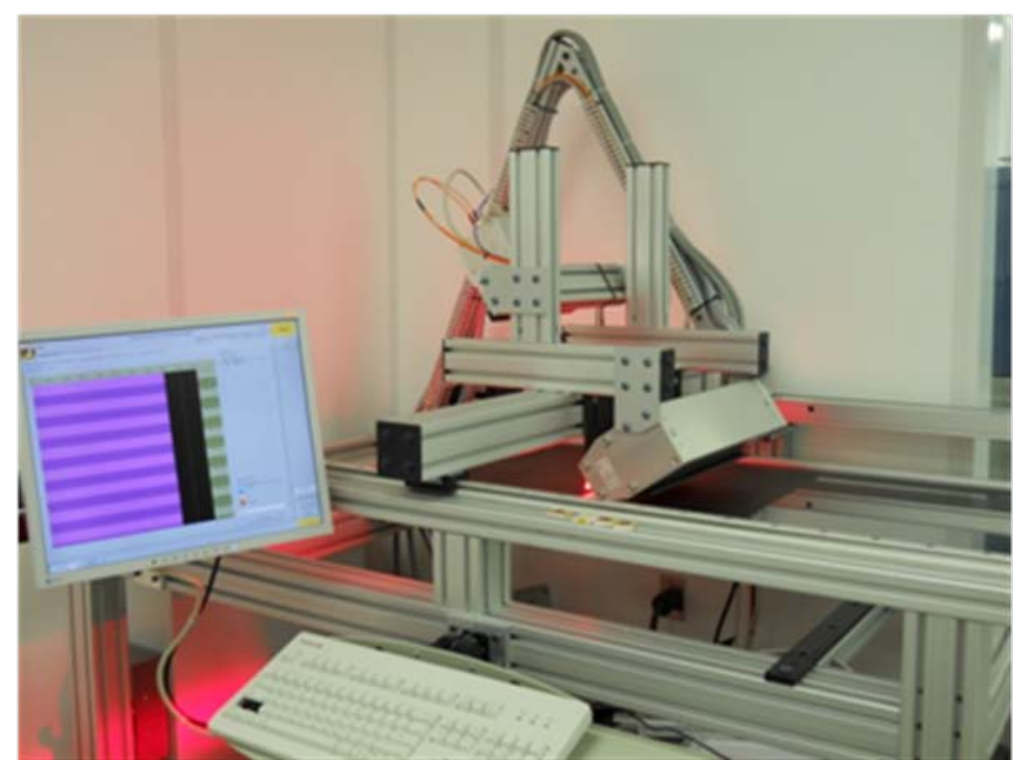

Figure 11 - The Inspection system referred to in the text as the Dr Schenk System. fibers and smudges. The defect count is recorded and tabulated.

Substrates used for experiments were 18" x 18" in size. Substrates are polished with Ceria in order to remove Tin specks, followed by thorough rinsing and washing at $130^{\circ} \mathrm{F}$. Later, organic contamination such as finger prints are removed by hand scrubbing with alkaline chemistry followed by rinse, pre-wash and wash at $130^{\circ} \mathrm{F}$. Cleaned substrates are inspected for defects using the Dr. Schenk camera system. It was found that polishing helped to remove tin specks and it is indicated by the lowered defect count recorded at the Dr.Schenk system. In addition, hand scrubbing of the substrate helped to eliminate organics and the difference can be seen in the defect images from Dr.Schenk.

Some work was also done using Ammonia in the washers to remove organics. The use of ammonia showed an improvement in the cleaning process.

As a part of defect analysis on the substrate, optical inspection of shorts has been carried out. Later, SEM/EDX analysis was conducted to understand the type of imperfection found at the origin of the defects. Most the defects seen were either metal contamination or organics located at various points within the thin-film stack. However, there were occasional substrate related defects such as tin specks.

\footnotetext{
${ }^{28}$ Tin specks are usually very small droplets of tin which are produced on the float line as a result of condensation of molten tin on the ceiling of the float bath. These then drip onto the glass ribbon as it is produced.

${ }^{29}$ See http://www.drschenk.com/ for details.
} 
Steady progress has been made on development of the cleaning process for the substrates, and this has focused on removing debris prior to the coating processes. Any remaining debris will

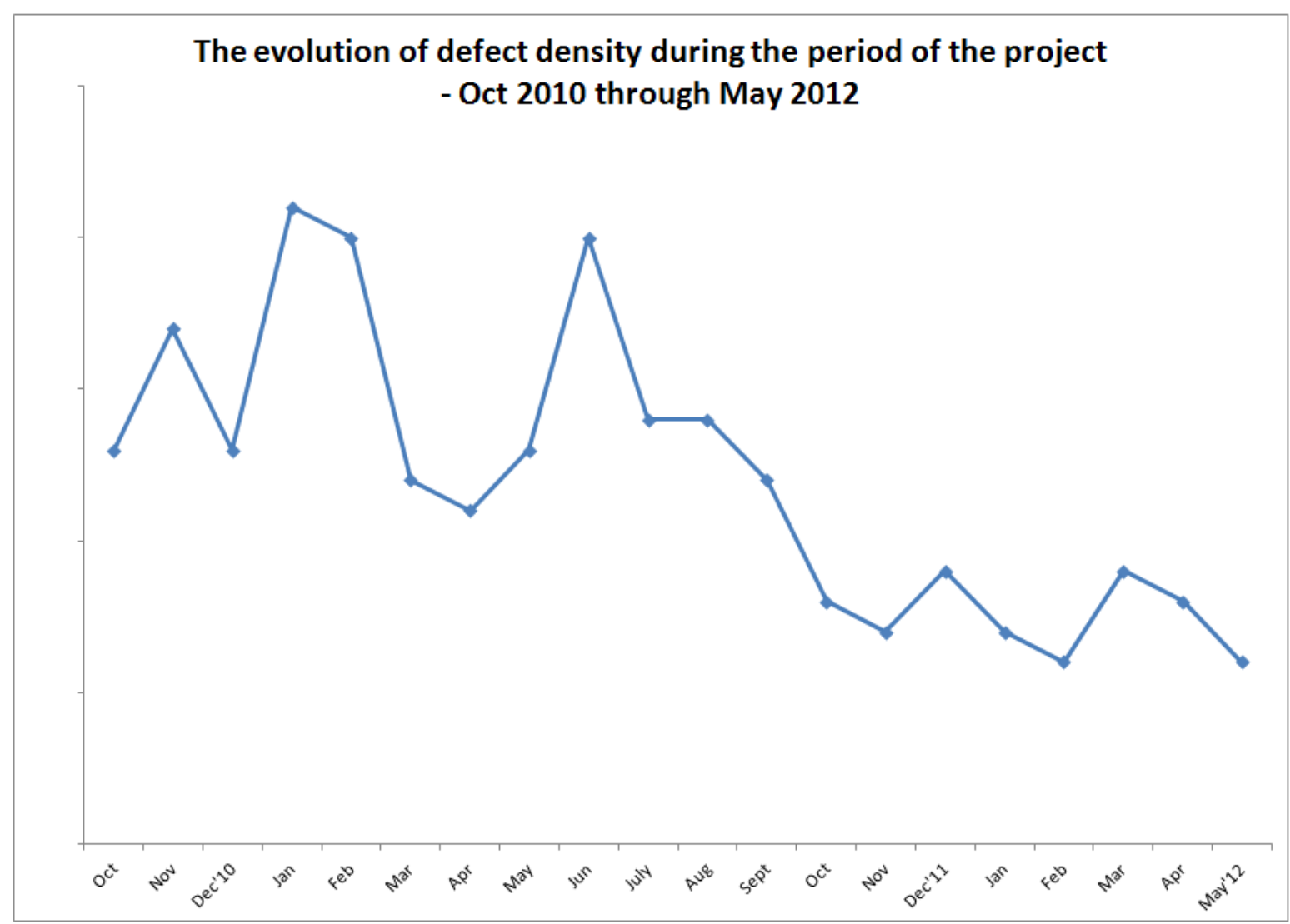

Figure 12 - Measured Defect Density during the course of the project showing incremental improvements as described in the text.

largely result in point defects, which manifest themselves as one of two major defects: shorts or non-coloring white dots. The observed quantities of these can be used to give an idea of the overall cleanliness of the process. We have seen significant decreases in the quantities of these types of defect as the project has progressed.

Figure 12 shows the incremental reduction in the number of point defects during the period of the project. This result shows the total improvement, which will have been achieved by a number of process enhancements, of which substrate cleanliness is one.

In order to allow elimination of the source of the defects, it is necessary to understand the origin of the contamination. In order to help with this, an analysis of shorts and white dots on experimental samples was conducted. These experiments were part of tuning the coating process input variables. Primary suspects were coating flakes. However after EDX analysis, most of the defects contained carbon, sulphur and fluorine on the bottom conductor. Some of the defects were from glass flakes. Therefore, no shorts were caused from coating particles but the root source is organic contamination in the process which is something which can be relatively easily controlled as presently the coating process itself is not optimized to reduce handling. 
The particle count data showed that tempered and non-tempered glass were similar in terms of numbers of defects. It is possible that there might be smaller defects $(<15 \mu \mathrm{m})$ that were not detected due to resolution of our system.

The deliverable for this subtask was a cleaning procedure resulting in a noticeable reduction in the rate of defect generation in EC devices. The continued observation of very low levels of defects in finished devices shows the efficacy of the cleaning process which has been developed during this project, clearly illustrating the successful accomplishment of this task.

\section{Task 3.2.2: Determine if Mobile Cations Impact EC Device Performance}

A consequence of using float glass is that there is a well known issue of sodium contamination of many different types of thin film devices arising from the substrate itself. This occurs when the thin films are deposited onto the float glass substrate, and is particularly noticeable when this is done at elevated temperatures. This task was intended to determine if barrier layers such as $\mathrm{SiO}_{2}$ or $\mathrm{Si}_{3} \mathrm{~N}_{4}$ are required between the glass and the device, and whether they can be fabricated with

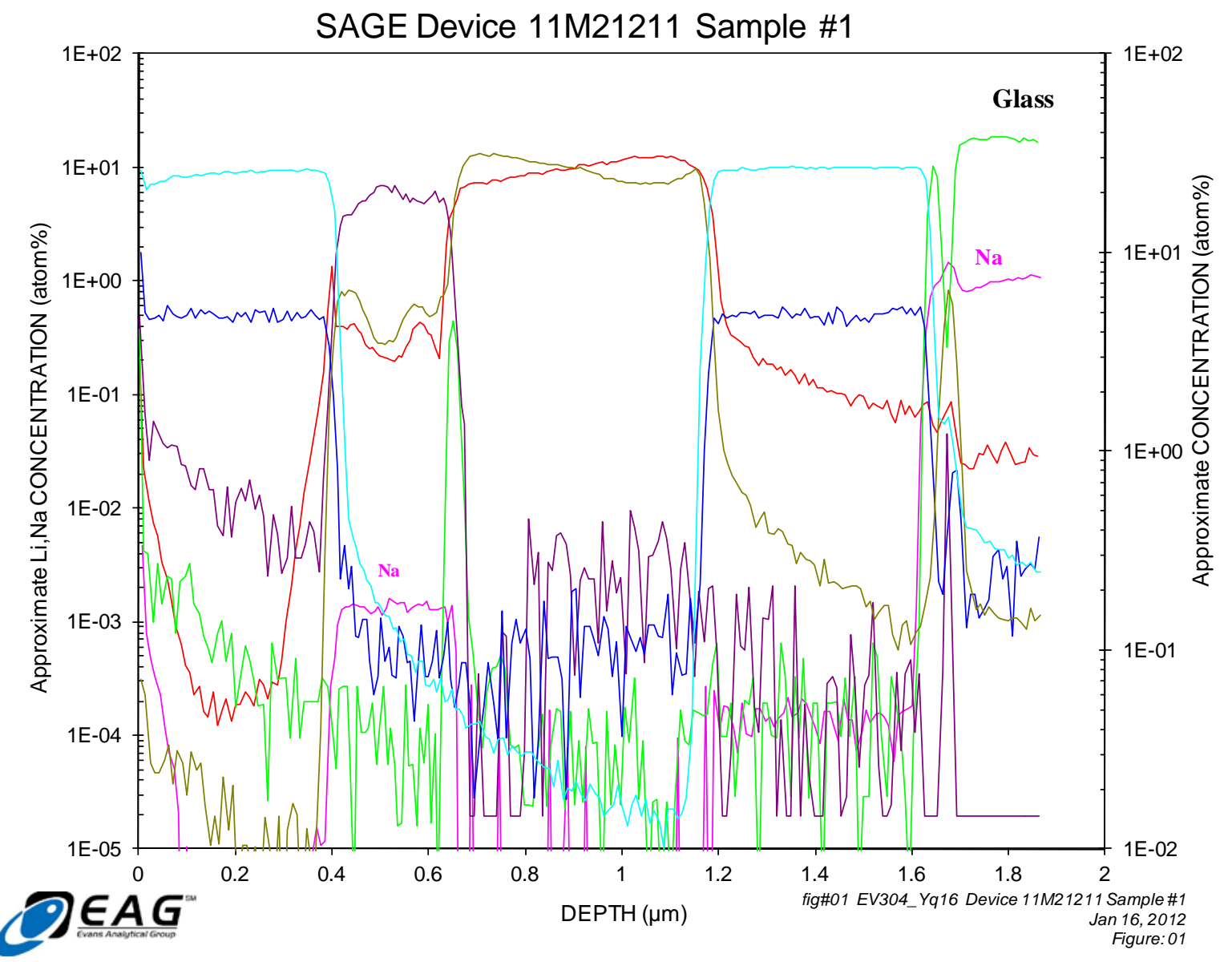

Figure 13 - Representative SIMS scan showing that the sodium does not penetrate the EC film stack at a meaningful concentration.

adequate ion-blocking properties. 
SIMS $^{30}$ analysis was performed on samples of the film stack deposited with barrier layers developed as part of Task 2.1, to determine if sodium ( $\mathrm{Na}$ ) diffused into the EC layers. The effectiveness of some of the as-deposited films as a barrier to Na migration was studied. Na was quantitatively analyzed. Several other ionic species were followed as layer markers.

The samples analyzed were produced using a representative film stack. Three devices were selected for SIMS with two spots on each device sent for analysis. For each of these analyses, the devices were profiled through the entire stack to find the $\mathrm{Na}$ concentration. In the EC layers the high concentration of dopant ions interfered with Na measurement slightly. The Na profiles were repeated with higher accuracy in these layers in an additional scan.

It is evident from Figure 13 that some of the as-deposited films are quite effective at preventing Na diffusion into the lower transparent conductor and EC films, particularly in this case where the subsequent films were deposited under special conditions.

Due to the efficacy of this barrier at blocking the Na migration, it is evident that running an experiment with low sodium glass would not yield a useful comparison. We therefore conclude that the undercoat layers as deposited here will provide adequate blocking of sodium in the EC device.

\section{Task 3.3: Increase the Sputter Rates for each of the EC Coatings}

This task aimed to improve factory throughput and therefore reduce the cost per square foot as a result of increased sputter rates from key target materials.

As a consequence of these higher sputter rates, the factory throughput will be increased and the cost $/ \mathrm{ft}^{2}$ will be reduced as fixed depreciation costs are spread over a larger volume of finished product. However, changing sputter rates can affect film structure, morphology, and stoichiometry. The task was therefore to increase rates yet retain desirable film properties.

Rotatable magnetron sputter deposition has become the most widely used coating technology for large area deposition. Many sputter targets are produced by plasma spraying, although this can lead to targets where the maximum applied power density is reduced compared to a fully dense single piece target. The failure mechanism of these targets can be delamination from the mandrel $^{31}$, particularly at higher deposition power.

\section{Task 3.3.1: Target Material Improvements}

This subtask was intended to improve target cooling, adhesion of target materials, and thermal expansion match between mandrel and target material to facilitate sputtering at higher power. The production of many targets by thermal spray processing is a state-of-the-art process, the success of which is due to several main factors:

\footnotetext{
${ }^{30}$ SIMS - Secondary Ion Mass Spectroscopy is a technique used in materials science and surface science to analyze the composition of solid surfaces and thin films by sputtering the surface of the specimen with a focused primary ion beam and collecting and analyzing ejected secondary ions. These secondary ions are measured with a mass spectrometer to determine the elemental, isotopic, or molecular composition of the surface.

${ }^{31}$ A mandrel is the support upon which the target material is deposited. These typically take the form of a tube made of material which is compatible with both the target material, and the process required to produce the target. It should also be thermally and electrically conductive, provide a convenient means for sealing at the ends, and also be non-magnetic.
} 
- Flexibility of target geometry and size allows for a wide range of shapes, diameters, and lengths;

- High deposition rates with good deposition thickness control and coating uniformity;

- Raw feed powder materials can be tailored to produce stoichiometric or nonstoichiometric compositions, thus allowing flexibility for specific applications.

Despite these benefits to the thermal spray process, limiting factors can still affect the coated target. The desired functionality of the sputter target can only be achieved by a well-designed coating that consists of a fully dispersed, dense, and uniform coating structure.

Mechanically blending dissimilar materials is quite useful, but it can provide many quality issues within the coating, including separation of particles in the plasma flame and overheating (evaporation) of lower melting point (MP) metals. This can result in increasing porosity levels and an inhomogeneous or uncontrolled coating composition.

A further problem is that the sprayed material can only be deposited up to a maximum thickness, leading to shorter lifetimes and therefore more regular maintenance intervals.

One solution to enable thicker, high density, uniform composition targets is to eliminate the spraying process completely and produce a single piece target. The following task discusses this development effort.

\section{Task 3.3.1.1: High Density Target Development}

At the beginning of the project, several of the targets used in the production process were produced by plasma spraying. In a plasma spraying process, the material to be deposited (feedstock) - typically as a powder, sometimes as a liquid, suspension or wire — is introduced into the plasma jet, emanating from a plasma torch. In the jet, where the temperature is on the order of $10,000 \mathrm{~K}$, the material is melted and propelled towards a substrate. There, the molten droplets flatten, rapidly solidify and form a deposit.

The plasma spray process is well suited to many materials, but can be problematic when binary compositions are utilized, particularly where the components have significantly different physical properties such as melting point and boiling point. As a result, it is difficult to maintain an accurate composition for such alloys, and so target production is challenging. Furthermore, as a result of the process of rapid solidification of small particles, there can be small voids, such as pores, cracks and regions of incomplete bonding.

As a result of these manufacturing limitations, there were some quality control issues with the EC product, and this task sought to address these issues.

A target manufacturing process has been developed to reduce the porosity of metals and increase the density of many ceramic materials, which improves the mechanical properties and workability of the material. 


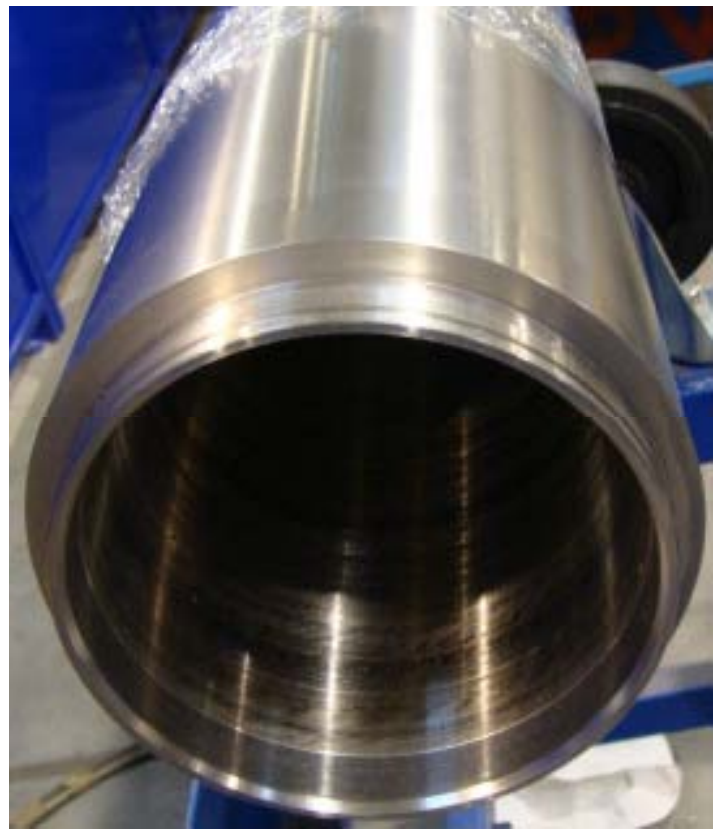

Figure 14 - The as received target tube
The major advantage offered by the high density process is to obtain much better compositional control over the currently used plasma sprayed targets. This is because the temperatures used do not approach the melting points of the materials, so any difference in physical behavior (i.e. vaporization at high temperatures) is not an issue. Additionally, the higher density of the target potentially leads to faster deposition rates, and the thicker target material will give longer times between maintenance.

A vendor was located who was willing to do some of the development with us for this relatively novel technique. After the first trial, the target failed due to critical internal stress, and so the processing temperature was lowered. This resulted in a successful second trial with reduced stress.

The target purchased under the project and fabricated using the improved process has been received. The initial inspection was excellent, and a photograph of the tube is shown in Figure 14. The target was installed, and has been successfully used in production.

Many further tasks were completed in order to get this target fully into production:

o The target had to be burned in and characterized as to composition and sputtering behavior;

o Standard operating procedures (SOPs) regarding the slow power ramp up had to be developed;

o Methodology was developed for understanding the effect of the power ramp up on target stability;

o Characterization of devices made with this target. 
A second iteration of the new target was received and installed in the Production Coater and underwent trials. This target had a slightly different composition than the previous one, and

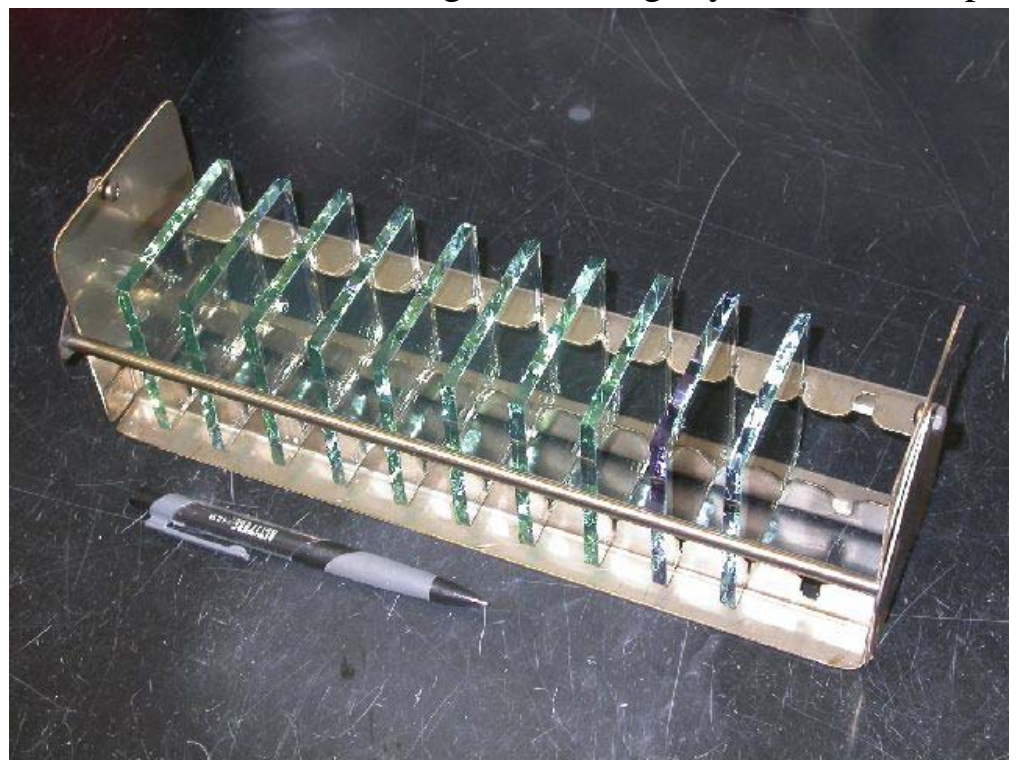

Figure 15 Rack of witness slides ready for electrochemical characterization. particular attention was paid to the surface finish, as any inhomogeneity will cause problems with the film uniformity and deposition characteristics.

The component materials in a multi-component sputter target typically erode at different rates. Initially, the more rapidly eroding material is sputtered away from the sputter target and the surface composition shifts accordingly. The sputter removal of the rapidly eroding material is balanced by depletion from the target surface, and a steady state is finally achieved.

It can take a significant time to achieve steady state, a process

known as "target burn-in.” Moreover, whenever the sputter target is removed from the sputter coater for maintenance, whenever a new target is installed, or whenever the process conditions of pressure, gas composition and sputter power are varied, the entire burn-in process must be repeated to achieve the new steady state.

We implemented a comprehensive program for electrochemical characterization to understand how the EC electrode materials evolve during the pre-sputter procedure, and to determine whether a definite electrochemical indicator could be found to efficiently detect the endpoint of the pre-sputter procedure. Figure 15 shows a rack of witness slides ready for characterization and Figure 16 shows the non-aqueous electrochemical cell used to characterize witness films. This program of detailed electrochemical measurements allowed us determine the

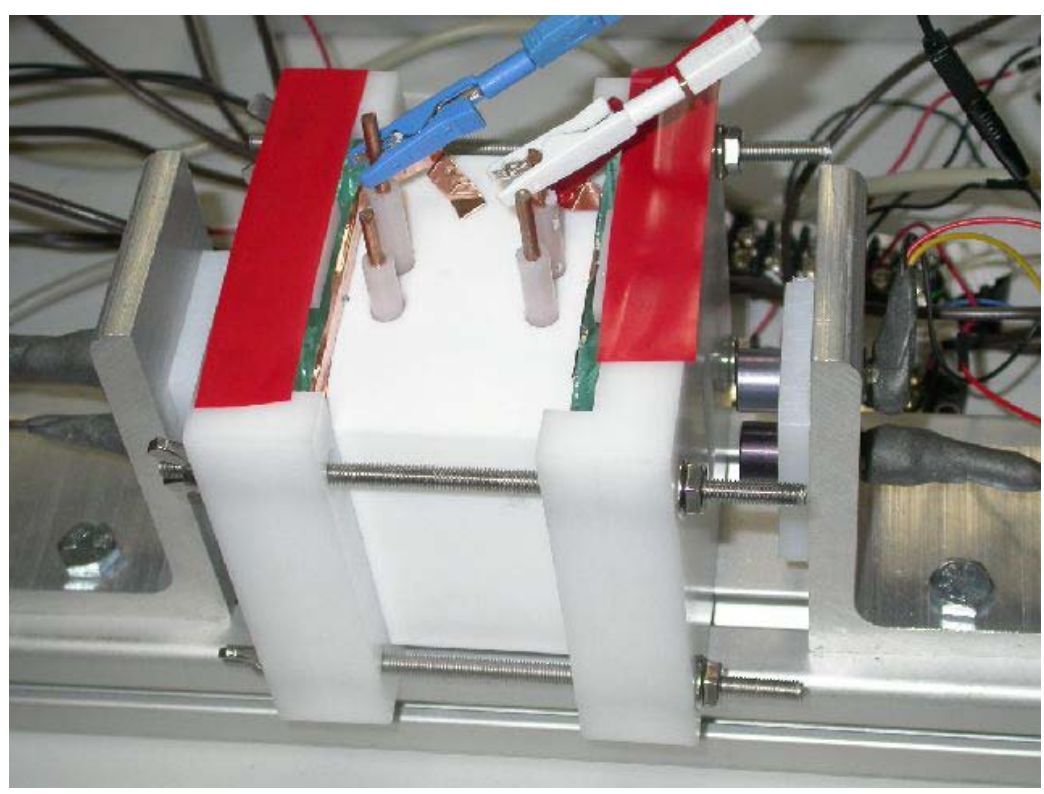

Figure 16. The non-aqueous electrochemical cell used for determining key properties of witness films. 
electrochromic efficiency of the active layers, in addition to their intrinsic transport properties, such as diffusion of counter ions, and charge transfer impedance.

We found that the electrochemical impedance of the sputter deposited EC material decreases from a few thousand Ohms to less than 500 Ohms as the pre-sputter process approaches the "process ready" condition. Also, we found that the electrochemical cycling current reaches a stable condition, and perhaps most unambiguously, the peak potential for the electrochemical reduction wave reaches a "process ready" limit.

In short, we now have new, sensitive, and robust monitoring technique for characterizing burn in, determining when burn in is complete, and monitoring process drift, to ensure that the process remains within process specifications.

\section{Task 3.3.1.2: Unique Dopant Target Development}

The issues which existed at the beginning of the project with the dopant ion target were primarily associated with the metallurgical bond between the target material and the mandrel. This tended to be patchy, and this could lead to areas which sputtered at a different rate, resulting in nonuniform film deposition. Additionally, this poor bond limited the maximum power which could be applied, thereby reducing the throughput which could be achieved.

An additional consequence of the manufacturing process was that the maximum thickness of target material was severely limited. This has the consequence of reducing the length of time between replacement of the target and further limiting overall throughput.

This task therefore aimed to develop specially fabricated targets capable of faster sputter rates and longer runs between replacement of the target used for the dopant ion as well as improving the bond between the target material and backing material.

SAGE worked with a vendor to manufacture several prototype targets for improved processing beginning in late 2010. We completed a series of DOE's in the first quarter of 2011 to identify a better approach to manufacturing the dopant targets for the project. Additional DOE work occurred in the second quarter of 2011 to further pursue observations from the initial experiments. In late March 2011, SAGE started a tooling process with the external supplier to produce the first of six prototype targets. The first tooling design review, conducted on April 12, resulted in several modification requests from SAGE, postponing the first prototype build by approximately 3 weeks.

The first process failed to achieve an adequate metallurgical bond between the target material and the mandrel surface. In the second approach, we completed a series of DOE's and range finding experiments to determine the best combination of surface, temperature and pressure to bond the target material to the backing material.

These DOE's were completed as planned and yielded confirmation of an improved process for the metallurgical bond of the target material to the target backing surface. The metallurgical bond was so strong that the target material tended to tear apart rather than separate at the interface point from the surface. The surface uniformity of the target was equally excellent.

Two full sized sputtering targets were constructed in September to the new process specifications and shipped to SAGE for testing in actual production in the CP-1 factory. These targets were installed in early October and exhibited high uniformity levels. Prototype 65-4 has exhibited no 
issues at all. Prototype 65-5 showed one small surface issue which was quickly cleaned up. Both targets were subjected to life testing in an actual production environment, with excellent results. Prototype 65-4 performed flawlessly in continuous life testing in the production environment

Overall, the development of the dopant target manufacturing process has been completely successful in that each of the problems identified at the start of the project have been eliminated.

\section{Task 3.3.2: Understand Higher Deposition Rate Film Properties}

As a natural consequence of higher sputter rates, factory throughput will increase and the cost $/ \mathrm{ft}^{2}$ of EC devices will be reduced, because fixed depreciation costs are spread over a larger volume of coated glass. However, changing sputter rates can affect film structure, morphology, and stoichiometry. The task was to increase rates yet retain desirable film properties, thereby maintaining yields.

In order to facilitate cost reductions, designed experiments (DOEs) to find the optimum process space where rates can be increased without changing film structure or performance, were carried out.

It is necessary to ensure that the film structure and behavior is maintained as deposition rate is increased, and it is well known that methods for increasing the deposition rate, such as increasing the power or increasing the amount of Ar in the sputter gas mixture, are likely to have an impact on the composition of the sputtered film.

Experimentation upon the deposition of the lower transparent conductor has shown a very interesting and complicated relationship between deposition conditions - including the deposition rate - and the properties of the EC device, and in particular the switching characteristics. It should be pointed out that this is not a direct consequence of the anticipated variation introduced by changes in the sheet resistance of the lower transparent conductor which can be characterized as 'in-plane' characteristics - rather the changes are seen in effects which are 'through' the device, i.e. perpendicular to the film layers.

However, extensive experimentation has revealed certain techniques for maintaining film properties and device performance as the sputter rate is increased. As an example, the deposition of the lower transparent conductor was increased by almost a factor of two, but the device functionality remained unaffected.

The indications are that given the activities discussed in Task 3.3.1.2 as well as other work associated with increasing the throughput, it is anticipated that the actual throughput of the production process could be increased in line with our predictions, resulting in significant cost reductions as anticipated.

\section{Task 3.4: Better Process Controls}

Almost all sputtering processes will drift to some extent during the life of a target, or even during a run. As a result, optical monitoring - usually transmission - of the deposited film is used to maintain the process within acceptable limits.

At the beginning of the project the production coater relied on simple transmission measurements where white light was brought into the coater via an optical fiber, passed through 
the glass under test, and returned to the outside via another fiber. It was then dispersed using a miniature spectrophotometer to generate a transmission spectrum of the coated glass. This spectrum was then displayed to the coater operator, where it could be interpreted and allow adjustment of the coating parameters. This technique is perfectly acceptable for non-absorbing films, because the interference fringes can be unambiguously interpreted to yield thickness and optical constants for a given film.

For highly absorbing films, as appropriate here, it is difficult to characterize the optical properties unambiguously, so it was proposed to develop a different monitoring scheme to facilitate increased automation and hence lower costs. The deliverable for this task was to be a prototype monitoring system and algorithms to provide closed-loop control of the coating process. We anticipated that the system would be implemented on the present production equipment in a limited way, but fully in the next large scale manufacturing plant.

\section{Task 3.4.1: Develop In-line Monitors for Critical Processes}

The first step in this process was to develop a capable monitor.

This was accomplished using off the shelf components which were readily available. Several systems were constructed and installed in the coater after critical layer deposition zones.

Software was written to display the measurements, control the calibration procedures and log the data to a SQL database.

This task has therefore been completed successfully.

\section{Task 3.4.2: Develop Algorithms to Enable Closed-loop Control of the Process}

At present, sputtering processes rely on the intervention of a human operator to make the necessary adjustments to stay within acceptable limits. We proposed to automate the process by developing algorithms and controls to eliminate the routine intervention of the operator, with an anticipated price reduction due to reduced labor.

The task can therefore be broken into the following components:

1. Acquire the relevant data (Read)

a. Compare with the accepted values,

b. Determine whether action is necessary and

c. Record data for future reference

2. If appropriate change the relevant setting to change control point (Write)

The software for reading the PLC and recording the data (Items 1, a, and c) is complete, and currently in use as a stand-alone data logging and monitoring application.

Item $1 \mathrm{~b}$ is somewhat complicated depending on the exact process, and requires advanced knowledge of the particular coating process. This will be addressed on the new high volume production equipment when it comes on-line.

Item 2 has also been demonstrated on the current equipment. A prototype monitor system and algorithms to provide closed-loop control of the coating process was developed, although this 
was not implemented due to the complexity of the coating process studied here. We anticipate implementation on the new high Volume Production facility.

The work done here has demonstrated the viability of this technique, although it has been fully implemented yet.

\section{Summary of Progress Made in Task 3}

The objective of Task 3 was to reduce the cost of the present electrochromic system, first by replacement of the expensive glass substrate and then by a series of process improvements. The preceding discussion shows that each of these objectives has been adequately met.

The replacement of the substrate with more readily available - and therefore significantly cheaper - float glass, had a number of possible technical implications (a) the surface cleanliness may have led to increased levels of defects, and the high sodium content may have produced undesirable issues with the device performance. The work carried out here to produce a high quality cleaning process, along with analytical work to investigate the sodium issue, has shown that neither of these should be future problems.

Increases in the deposition rates of several of the materials in the EC production process have been demonstrated. This has entailed a significant effort in materials development coupled with sputter target improvements. Along with this, we have demonstrated that the device functionality will be retained as the deposition rate of these films is increased, providing the opportunity for a particular piece of production equipment to coat glass at a higher rate. This will obviously lead to lower production costs.

Finally in this task, we have demonstrated better process control is feasible by putting in place all the necessary tools such as monitors and software to allow closed-loop control of the coating process. This will have the benefit of improving yields, as it will remove the sometimes flawed decision making intervention of the human operator, and improve the response time of such changes.

\section{Task 4: Further Reduced Cost and Lower U-value}

The U-value is a measure of the conductive heat energy transferred through an IGU. In the EC stack, one variable that impacts the U-value significantly is the emissivity ${ }^{32}$. If the emissivity can be reduced, heat loss from windows especially in northern and mixed climates, will also be reduced. It is desirable to maintain a high SHGC as U is reduced. The deliverable for this task was to be demonstration of a fully functional EC device using the elements described in Tasks 4.1 and 4.2 .

\section{Task 4.1: Lower the sheet resistance of the top transparent conductor:}

Anti-reflected metallic thin-film stacks are common practice for today's low-e glazings, and the sheet resistance can be as low as 2 Ohms/sq. Conservative estimates suggest that a reduction in sheet resistance of a given layer to $3.5 \mathrm{Ohm} / \mathrm{sq}$. can be achieved. The resulting lower emissivity should yield U-values for the EC devices equivalent to the best double pane low-e constructions. In this task, we proposed replacement of the TC with an alternative stack.

\footnotetext{
${ }^{32}$ The ratio of the radiation emitted by a surface to the radiation emitted by a blackbody at the same temperature.
} 
An optimum EC stack would include anti-reflected metallic films for both the top and bottom transparent conductors, but this will involve significant process developments.

As this work progressed it became increasing difficult to incorporate such a target - and the cathodes associated with the anti-reflecting layers - in the production coater, due to some of the reconfiguration which had to be done to meet some of the goals in the other tasks, as described earlier. As a result, we have not been able to fully complete this task, but we have done enough work in the areas of the support tasks to satisfy ourselves that the approach is feasible.

The work which was done to address this issue is summarized below.

\section{Task 4.1.1: Optimizing Optical Properties}

As discussed earlier in Task 2.3, optical data were generated for individual layers, and fitted to physical representative models. Optical models of the complete stack were generated with a view to understanding the effect of alternative top transparent conductors.

As part of this effort, we purchased two licenses of the optical modeling package, $\mathrm{CODE}^{33}$.

\section{Task 4.1.2: Assure Compatibility with EC Device Layers}

A series of DOE studies were to be carried out to optimize processing parameters for devices with the alternative. This will involve process temperatures, doping levels and layer thicknesses, to ensure that the film layers were compatible with the EC stack.

As described earlier, it was not possible to make complete stacks with the alternative top TCO.

The work in this area has been limited, but the indications are that given the correct coater configuration, EC devices with the alternative systems both top and bottom are achievable.

\section{Task 4.3: Determine U-values of Improved EC Windows}

We planned to work with a external firm to carry out optical measurements of the film stack and then thermally model a variety of potential window configurations including an additional low-e film on surface 3. This would allow us to choose the lowest cost, best performing solution to yield devices with the best possible U-value.

We have not been able to generate a sample with the alternative low-e top transparent conductor, but the modeling work has been possible, and a general framework for producing the desired result has been determined. This approach requires measurement of the emissivity of the top transparent conductor, and then a decision is made based on this as to whether the additional low-e coating on surface 3 is required. The application of this additional low-e is possible via a number of different approaches, including sourcing the coated glass externally, coating it internally, or applying it via a flexible after market film product.

\section{Summary of Task 4 Progress}

Although the overall aim of producing a device with an alternative top transparent conductor was not met, work on several of the sub-tasks has given us confidence that this goal is fully

\footnotetext{
${ }^{33}$ W.Theiss Hard- and Software, Dr.-Bernhard-Klein-Str. 110, D-52078 Aachen, Germany. Web: www.mtheiss.com email: theiss@mtheiss.com Phone: +(49) 2415661390 Fax: +(49) 2419529100
} 
achievable with the correct coater configuration. It was not possible to set-up the coater to be able to do this because of the competing requirements of (a) the present production process, and (b) the other tasks of this project. Given the limited number of available cathode positions in the coater, it was simply impossible to make room for the new targets. It has been found that it is very difficult to transport a partially completed device to an external vendor for application of a top transparent conductor, so this was not attempted here.

However, we did make some progress on the task whereby the optical modeling of the materials required to generate a model useful for predicting optical properties such as color, reflection and transmission, was completed. Additionally, we saw that it was possible to produce devices using the new lower transparent conductor.

\section{Task 5: Ongoing Durability Testing of New/Improved Device Structures}

SAGE has accelerated testing facilities that include simulated solar irradiation, temperature cycling, temperature/humidity exposure, and rapid EC cycling, which will be used to qualify new process developments as they occur, to ensure no loss of performance. A deliverable here was a report of the reliability studies described above, showing that the changes carried out have had no detrimental effect on the durability.

The purpose of this effort is to establish clear limits for voltage-induced degradation in the current production process, as a baseline to evaluate future changes.

An initial, brief round of experiments aimed to establish what sorts of degradation mechanisms are associated with excess voltage stresses were carried out. Devices generated as part of the project continue to cycle and the degradation mechanisms characterized. As improvements are made to the process, characteristic devices are added to those undergoing long-term cycling.

These representative samples of the devices produced during the project were subjected to the durability testing which is a routine part of the SageGlass manufacturing process. This includes a room temperature cycling process, where the devices are colored and bleached repeatedly, as well as a simulated solar soaking process, where devices are colored and bleached under illumination equivalent to one sun. In addition, there are a number of other tests carried out to prove out the durability of the EC system itself, including stresses aimed at testing the IGU fabrication, the bus bar adhesion to the films and the strength of overall glazing.

All of these tests have been carried out, and the devices have shown equal if not superior performance to the existing technology in terms of the durability. Considering first the cycling results, we see a slight decrease in the clear state transmission with cycling, but this is normal for the present technology. The leakage current is not significantly changed and dark state transmission remains relatively consistent throughout both cycling and solar soak.

In conclusion, the changes made during this project have had no detectable effect on the excellent durability of the SageGlass product.

\section{Discussion and Conclusion}

The overall goal of this project was to improve the energy performance and lower the cost of dynamic electrochromic (EC) smart windows for residential and commercial building applications. This project was clearly aligned with the DOE objective of zero energy buildings 
(ZEB) with high performance windows that provide thermal and daylighting benefits offsetting other building energy uses.

This proposal addressed two major areas: lowering the price and improving the energy performance of EC glazing. The goals for these activities were: (i) to improve the SHGC range, (ii) reduce the price of EC windows by utilizing lower cost materials, (iii) lower the U-Value, and (iv) ensure the proposed changes had no detrimental effect on the durability of the window.

It was proposed to achieve these objectives using a combination of materials development and economies of scale facilitated by increased production volume. The materials developments were challenging but as the preceding discussion has shown, they were largely achieved, and the cost reductions were modeled on the adoption of low-e glass: a technology with a similar market structure.

The project was divided into four main areas:

1. Improvement of the Properties of the EC device by material enhancements (Task 2);

2. Reduce the cost of production by improving the manufacturing processes (Task 3);

3. Further reduce the cost by significant modifications to the structure of the device (Task 4);

4. Ensure the durability of the EC device is not affected by any of the changes resulting from these activities (Task 5).

A detailed description of the activities carried out in these areas has been given in this report. We have seen that we have completed Tasks 2 and 3 fully, and the durability of the resulting device structure has been unaffected. Some of Task 4 was not carried out because of difficulties with integrating the installation of the required targets into the production coater due to external constraints not related to this project. We saw that the durability of the devices produced as a result of this work was largely unaffected by the changes made here, and so we can conclude that the objectives of Task 5 have also been fulfilled.

As a result, the future production of EC windows has benefited greatly from this work. We anticipate higher volumes and better yields for devices with an improved EC performance from the new high volume manufacturing facility currently under construction at SAGE.

\section{Technology Transfer Activities}

There was one patent application which derived directly from this work, and the details of this are outlined in form DOE F-2050.11 submitted separately.

There were no other technology transfer activities associated with this project. 


\section{Bibliography and References Cited.}

1. Lee, E S. Energy Performance Analysis of Electrochromic Windows in New York Commercial Office Buildings. Lawrence Berkeley National Laboratory. Berkeley, CA. : s.n., 2002. LBNL-50096.

2. N. Sbar, M. Badding, R. Budziak, K. Cortez, L. Laby, L. Michalski, T. Ngo, S. Schulz, K. Urbanik. Solar Energy Materials \& Solar Cells. 1999. Vol. 56, p. 321.

3. requirements, Reference for market research meeting customer.

4. C.G.Granqvist. Handbook of Inorganic Electrochromic Materials. s.l. : Elsevier B.V., 1995. ISBN: 978-0-444-89930-9.

5. NREL. Durability Test results (Private Communication.)

6. RF-superimposed DC and Pulsed DC Sputtering for Deposition of Transparent Conductive Oxides. Stowell, Michael, et al. 2007, Thin Solid Films, Vol. 515, pp. 7654-7657.

7. Lee, Se-Hee, et al. Electrochromic Counter Electrode. 6859297 USA, February 22, 2005.

8. Pilkington Optiwhite Datasheet.

9. Projected Benefits of Federal Energy Efficiency and Renewable Energy Programs. March 2005. NREL/TP-620-37931.

10. PNNL. Market Diffusion Data.

11. Inc, Lutron Electronics Co. Technical White Paper, P/N 367-1548.

12. Projected Benefits of Federal Energy Efficiency and Renewable Energy Programs. s.l. : NREL/TP-620-37931, March 2005.

13. D.B.Elliott, et al. Methodological Framework for Analysis of Buildings-Related Programs: The GPRA Metrics Effort. Richland, WA : Pacific Northwest National Laboratory, 2004. PNNL-1467.

14. Werthwein, Per. Shedding New Light on UV and Fading. s.l. : AFG Glass, 2005. http://www.showcasewindows.com/Websites/showcasewindows/Files/Content/167730/Shedding New Light on UV and Fading.pdf. 